\title{
The role of number of items per trial in best-worst scaling experiments
}

\author{
Geoff Hollis ${ }^{1}$
}

Published online: 9 July 2019

(C) The Psychonomic Society, Inc. 2019

\begin{abstract}
Best-worst scaling is a judgment format in which participants are presented with $K$ items and must choose the best and worst items from that set, along some underlying latent dimension. Best-worst scaling has seen recent use in natural-language processing and psychology to collect lexical semantic norms. In such applications, four items have always been presented on each trial. The present study provides reasoning that values other than 4 might provide better estimates of latent values. The results from simulation experiments and behavioral research confirmed this: Both suggest that, in the general case, six items per trial better reduces errors in the latent value estimates.
\end{abstract}

Keywords Semantic judgment $\cdot$ Best-worst scaling $\cdot$ Rating scales

Best-worst scaling is a tool for collecting human judgments or opinions. A set of $K$ items are shown to participants, who then choose the superior and inferior items along some latent dimension of interest. For example, participants may be shown sets of four words and be asked to choose the most-valenced of the set and the least-valenced of the set. Upon collection of judgments for many such sets with overlapping words, these data can be scored to assign items a value along the underlying latent dimension of interest (e.g., Hollis, 2018; Kiritchenko \& Mohammad, 2016).

Best-worst scaling has numerous desirable properties relative to other methods of collecting human judgments. First, the tool avoids the response biases endemic to rating scales due to being a rank judgment rather than an scalar judgment. Second, other ordinal judgment tools only require participants to make a single judgment (typically, the best item along some latent dimension). The requirement of having participants make a worst judgment provides additional information for differentiating items. Third, as compared to providing a full rank ordering, best-worst scaling only requires participants to make two judgments and is consequently much faster to make. Importantly, those two judgments nonetheless carry information about the relative rank of many of the pairwise

Geoff Hollis

hollis@ualberta.ca

1 Department of Computing Science, University of Alberta, Edmonton, Alberta, Canada combinations of $K$ items present. For example, when $K=4$ best-worst scaling provides relative rank information about five of the six possible pairwise combinations of items. For detailed accounts of the merits of best-worst scaling relative to other tools for collecting human judgments, readers are referred to Louviere, Flynn, and Marley (2015) and Hollis and Westbury (2018).

Best-worst scaling has seen two primary areas of application. The tool was originally developed within economics for estimating the perceived utility of various types of economic entities, including government spending policies (Finn \& Louviere, 1992), means for businesses to improve their competitive positioning (Soutar, Sweeney, \& McColl-Kennedy, 2015), and ethical practices of companies (Auger, Devinney, $\&$ Louviere, 2007). The fields of natural-language processing (NLP) and psychology have more recently begun using bestworst scaling to collect semantic norms for words, like with the example of valence above. In NLP, such norms have applications as features in sentiment analysis (e.g., Kiritchenko, Zhu, \& Mohammad, 2014) and in psychology they have applications in constructing models of lexical processing (e.g., Kuperman, Estes, Brysbaert, \& Warriner, 2014).

The economic and lexical use-cases differ in a methodologically nontrivial way. Applications of best-worst scaling to estimate perceived utility typically use the tool to collect judgments of a few dozen items at most. In contrast, use of bestworst scaling to collect semantic norms typically involves thousands or tens of thousands of items. Best-worst scaling can produce biased and noisy results when some items appear 
more often than others, or certain pairs of items are more likely to occur together than others. The two use-cases need to address this problem in different ways.

To remedy issues of undesirable variation in best-worst scaling, designs need to be employed that balance the appearance of items and item-pairs across presentation sets. Cases that involve dozens of items can accomplish this by borrowing procedures from experimental design, in which conditions and their interactions also need to be balanced-for example, through the use of balanced incomplete block designs (BIBD; Louviere et al., 2015). Because block designs increase in size combinatorially with the number of items to balance, they are not feasible for collecting semantic norms. Instead, many-item cases can use unbalanced designs along with randomization and many appearances of each item to reduce undesirable variation in best-worst data (see Hollis, 2018, for introduction an introduction to methodology in the many-item case).

For the remainder of this article, $N$ will be used to refer to the number of items over which best-worst scaling is being applied (e.g., dozens of items for perceived utility vs. thousands of items for semantic norms). $K$ will be used to refer to how many of those $N$ items appear on any given trial. Because small- $N$ cases (dozens of items) can utilize block designs to balance items, there is little decision making surrounding $K$; it is largely determined by the block design that fits the researcher's stimuli. However, since large- $N$ cases (thousands of items) cannot make use of block designs to balance items, researchers must make a deliberate decision about the size of $K$ to use.

Large- $N$ applications of best-worst scaling have exclusively used a $K$ of 4 . To our knowledge, the first piece of research introducing best-worst scaling for the large- $N$ case was Jurgens, Mohammad, Turney, and Holyoak (2012). No explanation was given why a $K$ of 4 was used, rather than $3,5,6$, or some other number. Subsequent large- $N$ research has likely reified the $K=4$ decision by how it introduces best-worst scaling. The common introduction is as follows (see, e.g., Hollis, 2018; Kiritchenko \& Mohammad, 2016): Best-worst scaling is a research tool for generating rankings among items. Its power comes from the fact that it provides information about the rankings of many pairs of items from only two decisions. Consider four items: A, B, C, and D. If A is chosen as best and $\mathrm{D}$ is chosen as worst, then information is provided about five of the six possible pairwise combinations of items. It is known that $\mathrm{A}>\mathrm{B}, \mathrm{A}>\mathrm{C}, \mathrm{A}>\mathrm{D}$, and that $\mathrm{B}>\mathrm{D}$ and $\mathrm{C}>$ $D$. The only pair for which no rank information is available is $\mathrm{B}$ and $\mathrm{C}$. This is much more information generated per decision than if, for example, participants decided between only two items at a time.

Large- $N$ applications of best-worst scaling have only been introduced with $K=4$, and during data collection the only value of $K$ that has been used is 4 . Both of these facts are likely creating biases to treat $K=4$ as a justified convention when, in fact, no justification has ever been given for this methodological decision.

There are rationally motivated reasons for considering using both larger and smaller values for $K$. As $K$ is increased, the amount of information generated per decision also increases. At $K=2$, information is generated about 1 out of 1 pairwise combinations of items, at $K=3$ it is 3 out of 3 , at $K=$ 4 it is 5 out of 6 , at $K=5$ it is 7 out of 10 , at $K=6$ it is 9 out of 15. Each increment of $K$ generates information about proportionally fewer of the pairwise combinations of items in a set, but more rank information overall. On this basis alone, researchers should want to use the largest $K$ possible, as that will generate the most rank information per decision.

There are at least two reasons why the value of the information generated by larger $K \mathrm{~s}$ might not be as great as the value of the information generated by smaller $K$ s. First, the larger $K$ might bias information to be about rank relations to extreme items. Participants are choosing the best and worst items among a set. Thus, all of the rank information generated would be about the items' relative relations to the most extreme cases. This could potentially leave the rankings between moderate values unknown. As $K$ gets larger, the chances of this happening would increase, because the chance of sampling an item with an extreme true value would increase. Second, it seems reasonable at face value to assume that past some point, increments to $K$ would increase the difficulty of the task and begin introducing noise into participants' decisions. Consider the cases of $K=100$ versus $K=2$. Much greater cognitive demands are placed on a participant in the $K=100$ case than in the $K=2$ case. These demands might lead to participants forgetting or neglecting some items or, depending on the strategy adopted for making their decisions, render the task intractable.

In sum, there are informational reasons to prefer larger values for $K$. However, there are also informational reasons why $K$ s past some threshold would also introduce noise and bias into the results. Finally, there are psychological reasons to believe that increasing $K$ would introduce noise into decision making. Currently, the value of $K$ that would optimally balance these constraints in large- $N$ designs is an open question.

\section{Experiment 1}

This experiment reports the results of simulating best-worst decisions under multiple parameter settings, including different values of $K$.

One of the choices that a researcher must make when collecting best-worst ratings is how to score their raw data. The most common way to score best-worst data is by tallying the number of times an item has been chosen as best and then subtracting from that tally the number of times an item has been chosen as worst (best-minus-worst scoring). These 
counts arrange items along a scale on which items consistently chosen as best and never chosen as worst are at one end of the scale, and items consistently chosen as worst and never as best are at the other end. Items that have a balance of best and worst choices, or which are never chosen, end up in the middle of the scale.

We will refer to two classes of scoring algorithms: count algorithms and predict algorithms. The above scoring algorithm is an example of a count algorithm; it organizes items along a scale by counting the number of times an event occurs (e.g., an item being chosen as best or worst). Other examples of count algorithms that can be applied to best-worst data include the analytic best-worst solution (ABW; Marley, Islam, \& Hawkins, 2016) and the David rank-scoring algorithm (David, 1987).

Predict algorithms instead score data by trying to predict the outcome of a pairing. Consider that a set of $K$ items can be further partitioned into some $J \geq K$ pairs of items. If A, B, and $\mathrm{C}$ are present on a best-worst trial $(K=3)$, and $\mathrm{A}$ is chosen as the best and $\mathrm{C}$ the worst, we can think of this set as three pairs, of which we know that $\mathrm{A}>\mathrm{B}, \mathrm{A}>\mathrm{C}$, and $\mathrm{B}>\mathrm{C}$. Predict algorithms take these pairs of items and attempt to predict which of the two will be ranked higher on the basis of a participant's response to a best-worst trial.

When considered in this way, best-worst data can be thought of as the results of a tournament. Players have been paired and play a match. One person wins and another person loses. The challenge of scoring best-worst data then becomes a question of how to derive the placement of each person in the tournament, on the basis of wins and losses. The problem of scoring tournaments is a well-studied area, with Elo (developed for ranking chess players; Elo, 1973) probably being the most widely known of such algorithms.

The key difference between predict algorithms (of which Elo is an example) and count algorithms is that predict algorithms do not assign scores based on best and worst choices, or wins and losses. Instead, predict algorithms assign scores based on how surprising the outcome of a pairing is. In chess, if a grandmaster plays a novice, it is highly likely that the grandmaster will win. After such an event occurs, the Elo ratings of the two players (i.e., their value along the underlying latent dimension) change only by a small amount; the outcome is not surprising and thus is not informative, given their current rating difference. However, it would be highly unanticipated, and thus highly informative, if the novice were to beat the grandmaster. If such an event were to occur, it would be indicative that the players' current relative ratings do not accurately reflect their relative skills. Thus, their Elo ratings would be adjusted by a much larger amount, to bring them closer together (the grandmaster's rating would go down, and the novice's would go up).

When applying count algorithms, a win (e.g., being chosen as best) carries the same weight, regardless of the difficulty of the competition. When a novice beats another novice, their score goes up by the same amount as when a novice beats a grandmaster. This turns out not to affect relative ranking if every player faces off against every other player, such as in a roundrobin tournament. Incidentally, this is something that balanced incomplete block designs partially accomplish when applied to small- $N$ cases of best-worst scaling. However, if players are only paired with a subset of other players - which must be the case, due to combinatorial constraints, in sufficiently large- $N$ designs - a player's rank can be distorted because they happened to have been paired with unusually tough or easy competition. Count algorithms do not mitigate this noise; predict algorithms do. Hollis (2018) introduced three prediction-based algorithms for scoring best-worst data: Elo scoring, value scoring, and Rescorla-Wagner scoring. Hollis (2018) and Hollis and Westbury (2018) provide simulated and empirical data that suggest prediction-based algorithms generally, but no always, provide more accurate estimates of an underlying latent dimension than do count-based algorithms in large- $N$ applications of bestworst scoring. However, the most appropriate scoring algorithm in a particular instance depends on the distribution of items across the underlying latent dimension.

The distinction between prediction-based and count-based scoring methods matters when considering the value of $K$ in best-worst experiments. As $K$ increases, the pairings for which information about wins and losses is available become increasingly biased to include extreme items. To continue with the tournament metaphor, the parallel is if all tournaments were designed so that each match must include at least one grandmaster or at least one poor chess player. This polarizes the informativeness of outcomes when the second player is of moderate rank; either the player loses to a grandmaster or wins against a poor player, and the outcome is mostly uninformative (which will happen most of the time), or the player beats a grandmaster or loses to a poor player, and the outcome is highly informative (which will happen very rarely). Count algorithms are insensitive to these differences, whereas predict algorithms will scale changes in a player's score on the basis of the informativeness of the outcome.

Inability to distinguish between high- and low-information cases should mean that count algorithms will generate noisier results as $K$ is increased, past some threshold. The specific value of that threshold is an empirical question. Recall that increasing $K$ also increases the total number of pairings for which information is available. This will mitigate to some extent the fact that the outcomes of those pairings will carry less information on average.

Predict algorithms should also generate noisier results past some threshold value of $K$, but overall predict algorithms should be more robust than count algorithms to these effects. This is because predict algorithms discriminate between informative and uninformative outcomes and scale changes in an item's score accordingly. 
We are thus led to the following set of predictions when considering large- $N$ applications of best-worst scaling: First, as $K$ increases, best-worst scaling will produce better estimates of the underlying latent dimension. This is primarily due to increases in the amount of pairs for which rank information is generated. Second, past some critical threshold of $K$, the accuracy of estimates will decrease. This is due to two causes: a reduction in the amount of information carried by pairs for which relative rank is available, and noise introduced by the increased cognitive demands of having to make decisions over larger sets of items. The final prediction here is that the critical $K$ threshold should be higher for prediction-based scoring algorithms than for count-based scoring algorithms. This is because prediction-based scoring algorithms discriminate between high- and low-information outcomes and scale the changes to an item's score accordingly, whereas count algorithms do not.

\section{Method}

The following simulation experiments were carried out using a methodology similar to that reported in Hollis (2018). Eight hundred forty items were generated so as to have true values distributed across an underlying latent dimension. Sets of size $K$ were generated from these items. Best-worst decisions were then simulated over these sets by choosing the item with the largest true value (best) or the smallest true value (worst).

Eight hundred forty items were used because this number is divisible by all of the numbers from 2 to 8 (inclusive). This property of divisibility allowed us to simulate results with all values of $K$ from 2 to 8 without the problem of some items occurring more or less than others, which would happen if the total number of items were not divisible by $K$; items can be grouped into 420 sets of $K=2,280$ sets of $K=3,210$ sets of $K$ $=4, \ldots$, or 105 sets of $K=8$.

For each condition of $K$, results were simulated for $1 N, 2 N$, $4 N, 8 N, 16 N$, and $32 N$ trials. Since $N=840$, those values are $840,1,680,3,360,6,720,13,440$, and 26,880. Trials were constructed on the basis of the best practices described by Hollis (2018): Each item should appear an equal number of times, and each item pair should occur only once. Note that these practices can be seen as a relaxation of the constraints of balanced incomplete block designs (BIBDs) that create combinatorial explosion as $N$ increases: Each item appears an equal number of times and also appears with each other item once. The software made available by Hollis and Westbury (2018) was used to generate trials and score results.

Four different distributions of true values were used: normal, uniform, exponential, and $F$. Parameters were identical to those used in Hollis (2018): Values were bounded between 0.0 and 6.0 for the uniform distribution, a decay rate of 1.0 was used for the exponential distribution, $d f_{1}$ was set to 100 and $d f_{2}$ to 10 for the $F$ distribution, and a $\mu=0.0$ and 휫 $^{2}=1.0$ were used for the normal distribution. These parameters were chosen because they all produce qualitatively different distributions, but in each case the large majority of values fall in the range of 0.0 to 6.0 (or -3.0 to 3.0 , in the case of the normal distribution).

Decisions were simulated under varying noise conditions. Prior to each simulated best-worst decision, some degree of random noise was added to each item's true value. Best or worst choices were then made on the basis of which item's true value, plus noise, was highest or lowest on that trial, respectively. Noise was sampled from a normal distribution with $\mu=0.0$, and 휫 was set to $0.0,0.5,1.0$, or 2.0 . The use of normally distributed noise in simulated judgments over a latent scale is supported by Thurstone's law of comparative judgment (Thurstone, 1927).

Item scores were derived from the simulated decisions using both count and predict algorithms. The following count algorithms were used: best-minus-worst, $\mathrm{ABW}$, and David rank scoring. The following predict algorithms were used: Elo, value, and RescorlaWagner. A summary description of each algorithm is provided below. Readers are referred to other sources for the complete formal description of each algorithm: David (1987) for the David scoring algorithm, and Hollis (2018) for the remainder of the algorithms. Derived scores were compared to the true values, using $R^{2}$ to assess quality of fit.

Elo scoring Prior to scoring, items were assigned identical initial values. Scores were adjusted on the basis of the pattern of wins and losses in the judged data. If an item won a pairing (i.e., was chosen as being higher-ranked than the other), its score was increased. The corresponding losing item had its score decreased. The larger the difference in current scores between the two items, the larger the adjustment after accounting for a pairing.

Value scoring An item's score was incremented toward 1 after a win and toward 0 after a loss. The degree of change in scores after a pairing was scaled as a function of the difference in current scores.

Rescorla-Wagner scoring Items were associated with win and loss events. An item's score was calculated as the ratio of its association strength to the win event to its total association to both events. Changes in association strengths were scaled as a function in the difference in current scores.

Best-worst scoring Item scores were calculated as the number of times an item was chosen as best, minus the number of times it was chosen as worst.

$\boldsymbol{A} \boldsymbol{B} \boldsymbol{W}$ scoring The analytic best-worst score was calculated as the logarithm of the ratio: one plus the unitnormalized best-worst score, over one minus the unitnormalized best-worst score. 

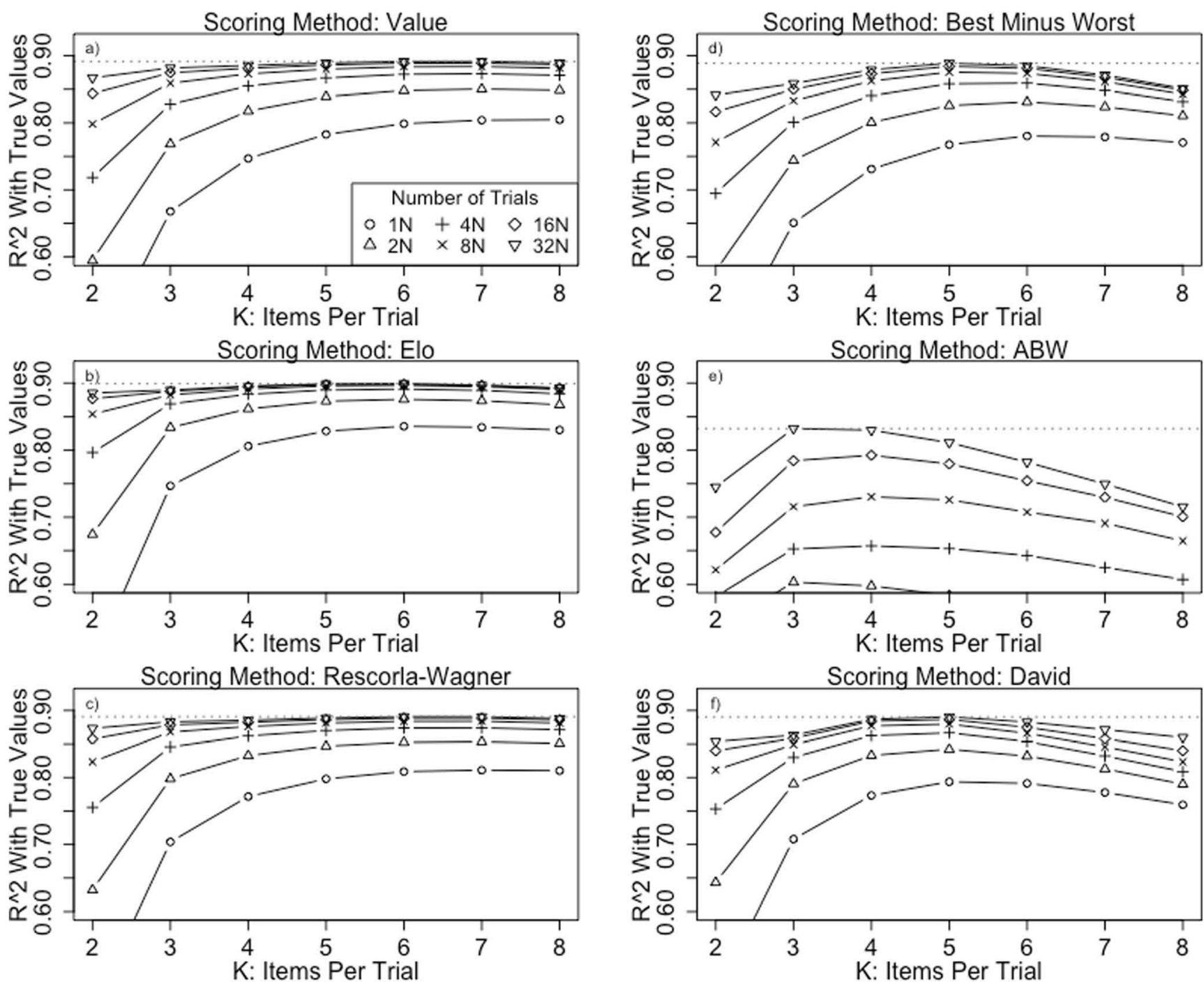

Fig. 1 Average fit $\left(R^{2}\right)$ between scores and true values for different values of $K$ and different numbers of trials. Results are displayed only for a noise level of 0.0 and are averaged across all four distributions of true values. Standard error bars are too narrow to be visible and are thus excluded from the figure. Panels a-c display the results for prediction-

based scoring methods (value, Elo, and Rescorla-Wagner, respectively), and panels d-f display the results for count-based scoring methods (bestminus-worst, ABW, and David rank scoring, respectively). Red dotted lines indicate the best-fit parameter settings in each panel

David scoring The score for a particular item, $J$, was the sum of the wins of other items that $J$ had defeated, minus the sum of the losses of other items that $J$ had been defeated by, plus $J$ 's own win count minus its own loss count.

A total of 100 unique simulations were run for each combination of true value distribution, number of trials, $K$, and noise. The results of each simulation were then scored with each of the six scoring algorithms. The derived scores were always compared to the underlying distribution of true values for that particular parameter combination.

\section{Results}

We started with an analysis of variance (ANOVA) to test for the effects of $K$ and for possible interactions of $K$ with other parameters. ANOVAs were conducted by entering the distribution, noise level, number of trials, and $K$ as variables. All two-way interactions were included, as well as all three-way interactions containing $K$. Variable levels were treated as categorical rather than continuous. Six ANOVAs were run, one using each scoring method's $R$-squared, with the true values as the outcome measure. All six ANOVAs revealed the same effects. The only difference was in the magnitude of those effects. We only report results for best-minus-worst scoring, the most commonly used scoring algorithm. 


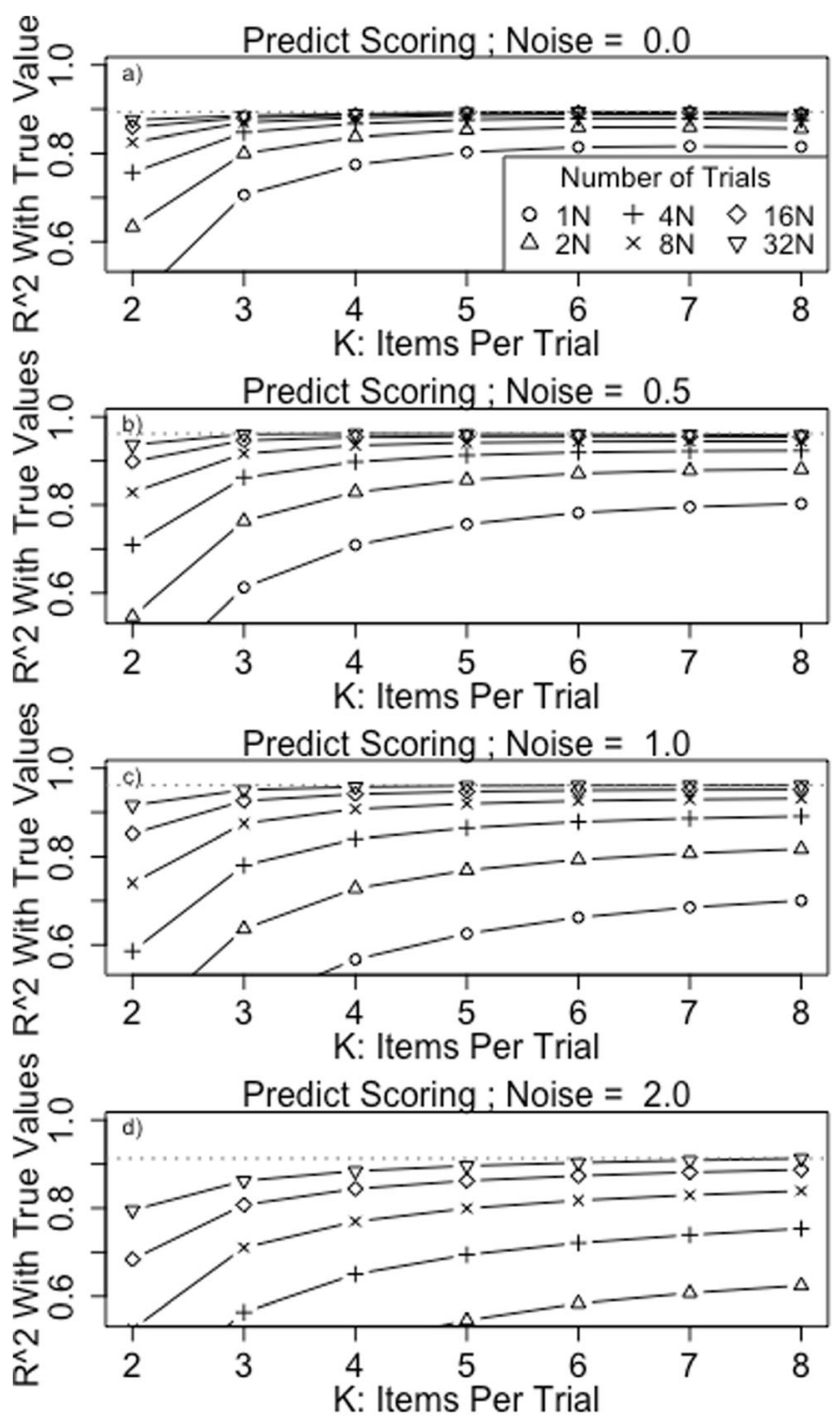

Fig. 2 Average fits ( $R$-squared) between the derived scores and true values for different values of $K$ and different amounts of judgment noise. Panels a-d display the results averaged over prediction-based scoring methods (value, Elo, and Rescorla-Wagner), and panels d-f display

Highly reliable effects were found for the underlying distribution of true values $[F(3,66843)=237,336, p=0],{ }^{1}$ noise level $[F(3,66843)=226,595, p=0]$, and number of trials $[F(3,66843)=481,578, p=0]$. These were all expected effects and have been documented before (Hollis, 2018). In short, the match between scores and true values depends on the underlying distribution of true values, how much noise is present in the judgment, and the amount of data collected. More to our interest was in the fact that there is also a highly reliable effect of $K[F(6,66843)=182,228, p=0]$, as well as highly reliable effects for all two-way and three-way interactions containing $K(F$ values ranging between $121, p=0$, and

\footnotetext{
${ }^{1}$ Of course, $p$ values can not actually be zero. Here, the convention $p=0$ is used for all cases in which the $p$ value was lower than the precision of our statistical software allowed for, $p<2.2 \mathrm{E}-16$.
}

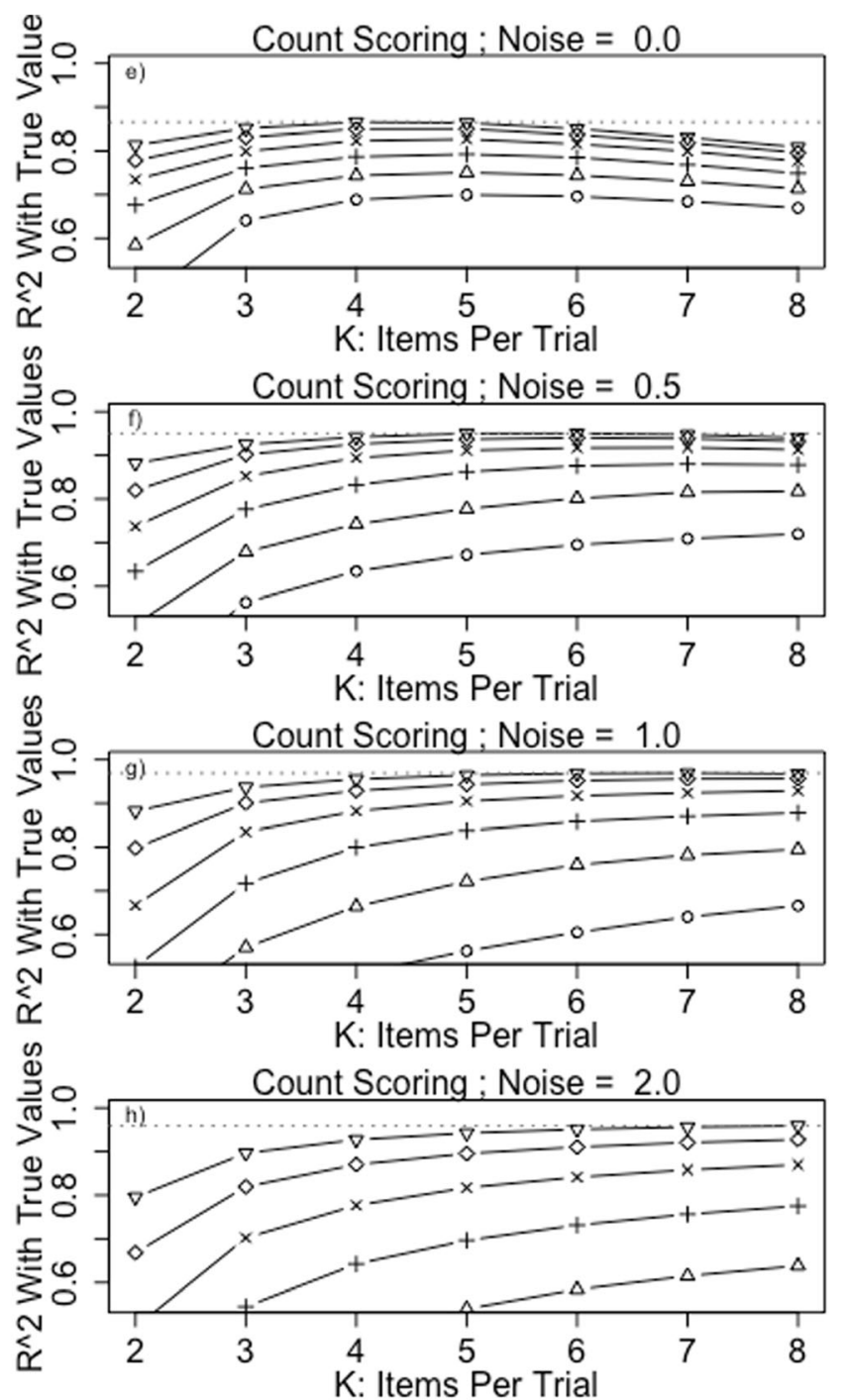

the results aggregated over count-based scoring methods (best-minusworst, $\mathrm{ABW}$, and David). Red dotted lines indicate the best-fit parameter settings, on average, in each panel

$6,917, p=0) . K$ has an effect on the quality of scores produced by best-worst scaling, and its effect depends on the underlying distribution of true values, the amount of noise in the data, and how many best-worst decisions are collected. These patterns were found for all six scoring methods, suggesting $K$ is a consideration regardless of how best-worst data are used to estimate items' latent values.

Noiseless judgments The average $R^{2}$ values between scores and true values, for different levels of $K$ and different numbers of trials when no noise is present in the data, are displayed in Fig. 1. Each panel displays the results for a different scoring algorithm. Standard error bars were too small to be visible in Fig. 1, and the $F$ values of $K$ 's main effect and interactions 

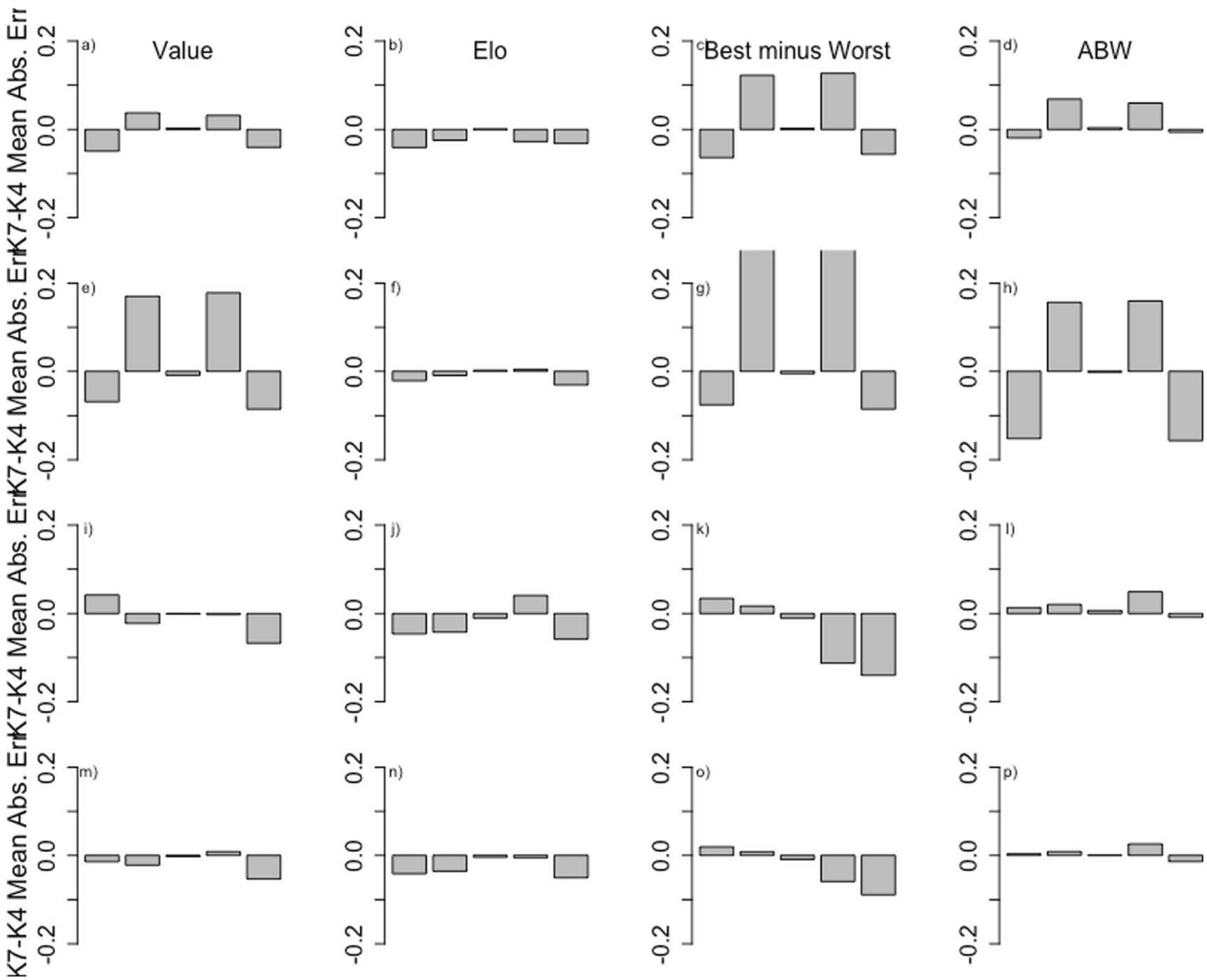

Fig. 3 Differences in mean absolute prediction errors for the cases of $K=$ 7 and $K=4$. Negative values indicate that $K=7$ has less absolute prediction error, on average. Each bar corresponds to one fifth of the data, from the 1 st-20th percentiles (left) to the 81 st-100th percentiles

were all quite large, so we make the assumption that all the visually identifiable effects involving $K$ are reliable.

As expected, increasing $K$ improved the quality of the observed fits up to a critical value. Beyond that value, further increases in $K$ reduce the quality of observed fits. Furthermore, also as expected, this effect is more pronounced for count-based than for prediction-based scoring methods.

Two effects involving $K$ bear further discussion. First, the critical value for $K$ depends on the scoring method used. For the analytic best-worst (ABW) solution, the optimal value for $K$ on average is 3 or 4 , depending on the number of trials data are collected for. For best-minus-worst scoring, the optimal value is 5 or 6 , and for David's rank algorithm it is 5 . For the prediction-based scoring algorithms, the optimal value of $K$ is somewhere in the range of 5 to 7 , depending on the number of trials for which data are collected.
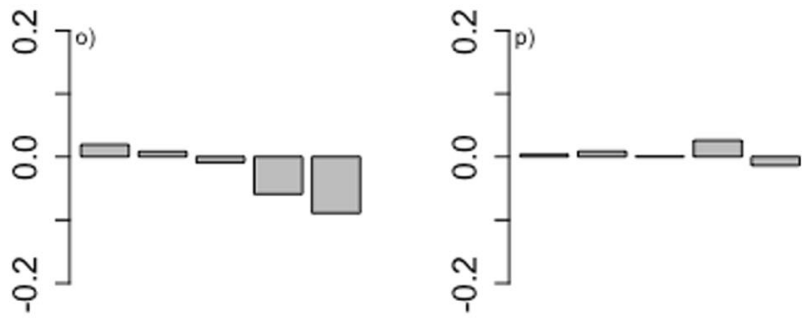

(right). The panel rows correspond to true-value distributions of normal, uniform, exponential, and $F$ (top to bottom). The panel columns correspond to the scoring methods of value, Elo, best-minus-worst, and analytic best-worst (ABW) (left to right)

The second effect we elaborate on, which was unanticipated, has to do with the near-equivalence of $K \mathrm{~s}$ as the number of trials is increased. For prediction-based methods, the quality of the estimates produced by different $K$ s begins to converge (at least in the range of $K$ s tested) when data for many trials are collected. For example, the quality of the fits produced by Elo (Fig. 1b) is much less sensitive to $K$ when data are collected for $32 N$ than for $1 N$ trials. The same is not true for any of the three count-based scoring methods, at least within the range of number of trials simulated in this experiment.

When no noise is present in judgments, the simulation results suggest that the convention of $K=4$ is not ideal in the general case. Larger $K \mathrm{~s}$ ( 5 to 7 ) produce better fits for prediction-based methods. $K \mathrm{~s}$ in the range of 3 to 6 may be preferred if counting algorithms are used. For best-minusworst scoring, which is the most commonly used scoring 

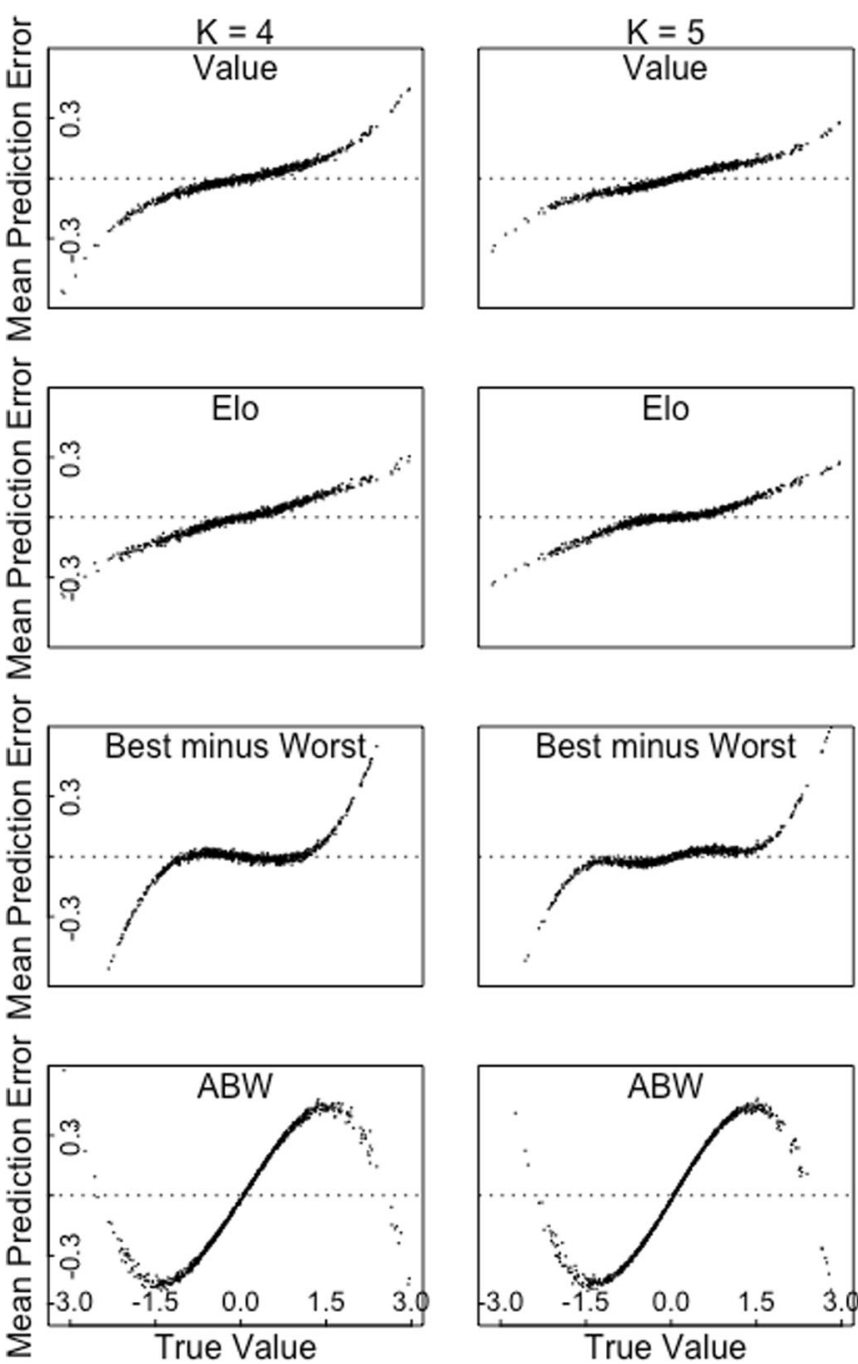

Fig. 4 Magnitudes of errors made by four scoring algorithms when scoring items with normally distributed true values. From left to right, the plot columns correspond to $K$ s of $4,5,6$, and 7 . From top to bottom, the plot rows correspond to the value, Elo, best-minus-worst, and analytic

algorithm, ${ }^{2}$ in no case tested was a $K$ of 4 optimal within a particular band of number of trials; the optimal value was always 5 or 6 .

Despite evidence that best-worst scaling produces data with high test-retest reliability (e.g., Kiritchenko \& Mohammad, 2017), the idealized circumstance of no noise in human judgments seems unlikely to occur in practice. Thus, we now turn to examining interactions between $K$ and noise levels, for which the previous ANOVA indicated strong interactions $[F(18,66843)=4,546, p=0]$.

Noisy judgments The qualitative patterns of interactions between $K$ and data noisiness were largely the same within a

\footnotetext{
${ }^{2}$ Note, though, that rarely does the scoring method that derives scores most strongly correlate with the true values.
}
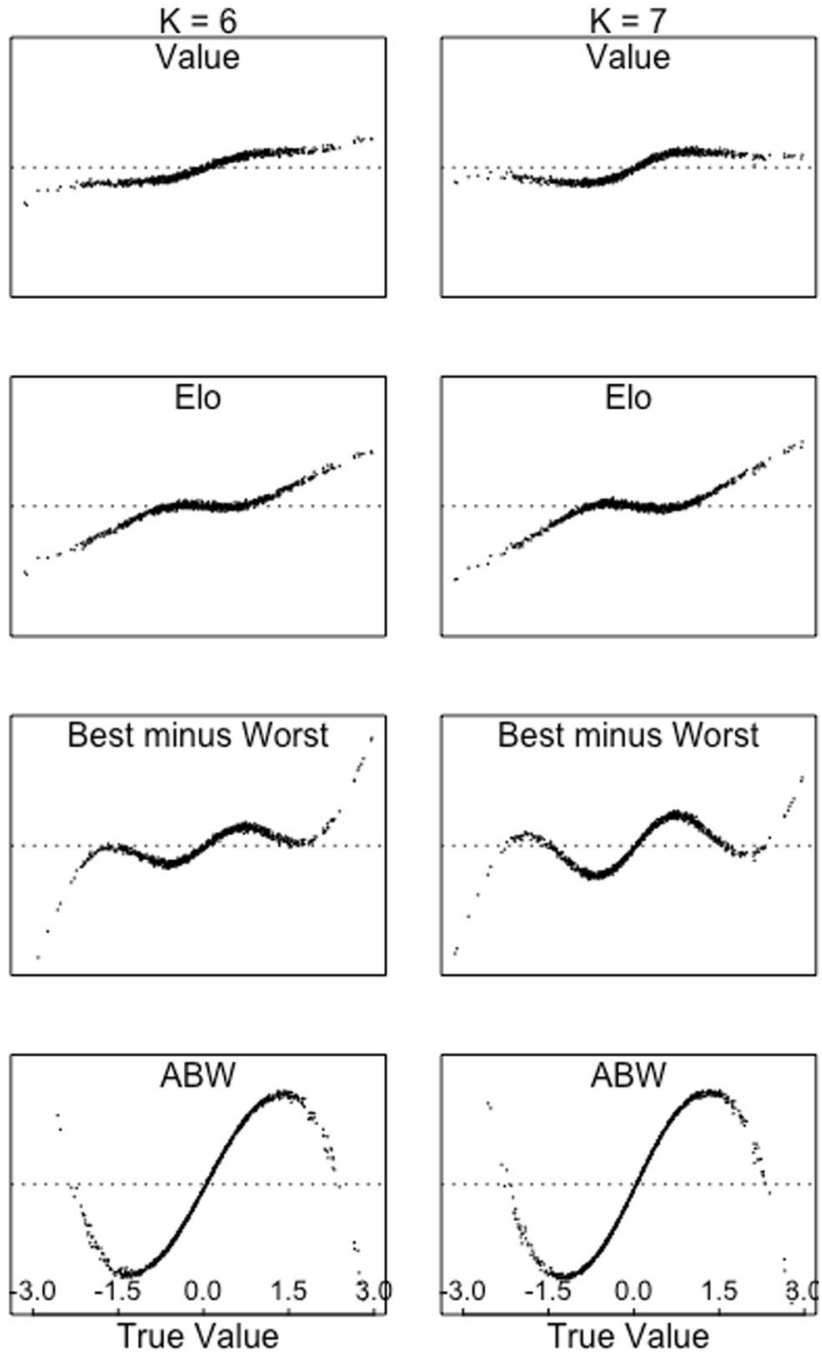

best-worst (ABW) scoring algorithms. The results are for the noise condition of 0.5 and are averaged over simulations with numbers of trials from $1 N$ to $32 N$

specific category of scoring algorithm (prediction-based vs. count-based). To reduce the complexity of figures, we now present results averaged over all scoring methods within a particular category. Such results are displayed in Fig. 2.

The most important point to note about Fig. 2 is that, as noise levels increase, the best-fitting results are produced by larger $K$ s. For noise levels of 1.0 and 2.0, a $K$ of 8 consistently produces scores most strongly correlated with the true values, regardless of the scoring method used. Hollis (2018) noted that within the present simulation framework, the types of semantic judgments that best-worst scaling is used to collect data for are best characterized by a noise level around 0.5 . Even at the noise level of 0.5 , the optimal value for $K$ is 6 or 7 , depending on the number of trials that data are collected for - greater than the $K$ of 4 that has exclusively been used to collect semantic norms. 

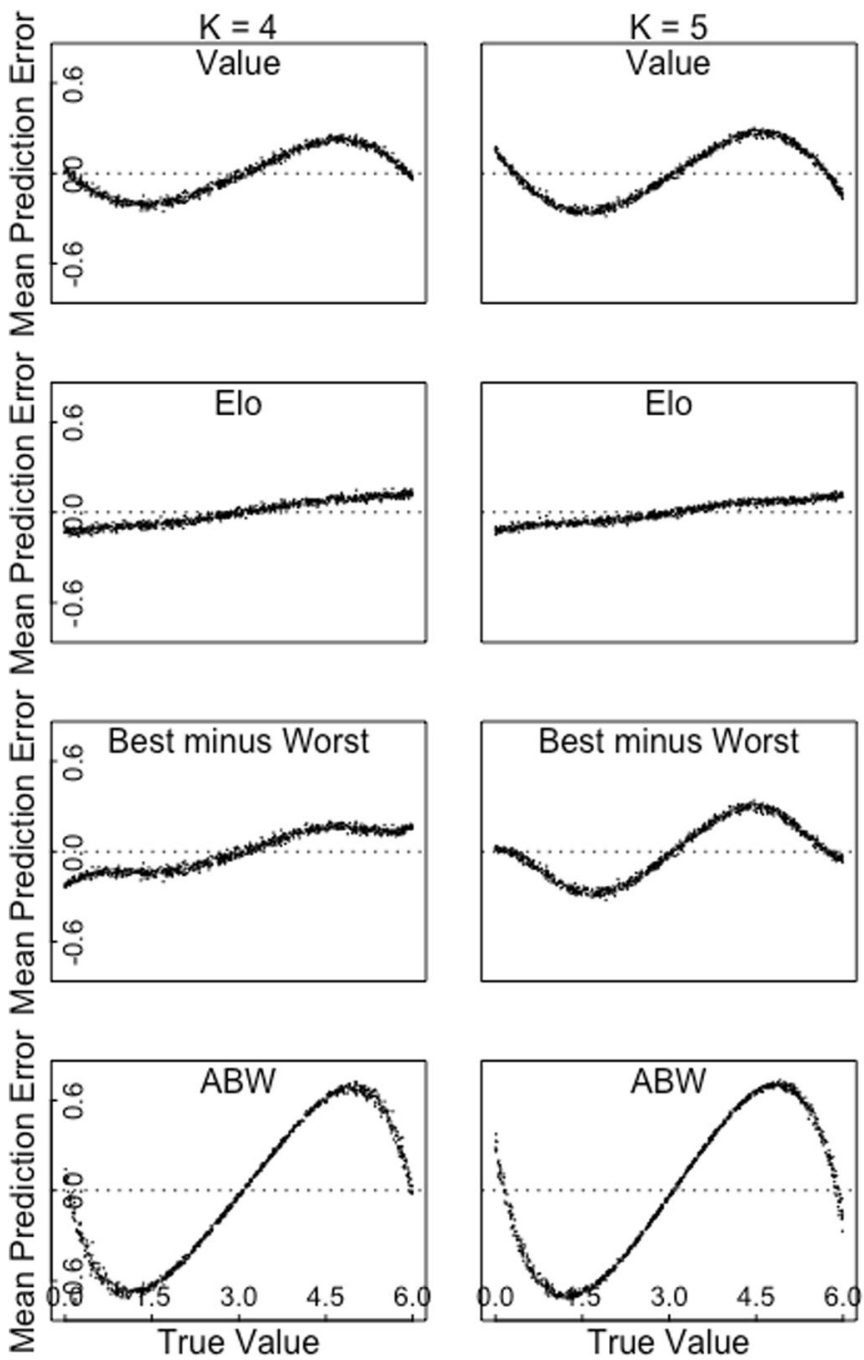

Fig. 5 Magnitudes of errors made by four scoring algorithms when scoring items with uniformly distributed true values. From left to right, the plot columns correspond to $K \mathrm{~s}$ of 4, 5, 6, and 7. From top to bottom, the plot rows correspond to the value, Elo, best-minus-worst, and analytic

Prediction errors as a function of $\mathrm{K}$ As $K$ is increased, more information becomes available in any particular best-worst trial, but that information is about rank relationships to more extreme items. One of the consequences of this is we should expect prediction errors to be non-randomly distributed across the range of true values and that the amount of systematicity should increase as $K$ increases. For instance, we might expect that as $K$ is increased, scores better predict true values of extreme items than they do for moderate items.

As an initial test of this hypothesis, we plotted the mean difference in absolute prediction errors for the cases of $K=$ 7 and $K=4$, for all four distributions averaged across all numbers of trials from $1 N$ to $32 N$. Only data for noise levels of 0.5 were used, and the results for RescorlaWagner scoring and David scoring are omitted. The rationale for these restrictions, along with using $K=7$ and $K=4$ rather than $K=8$ and $K=2$ is given below as part of a
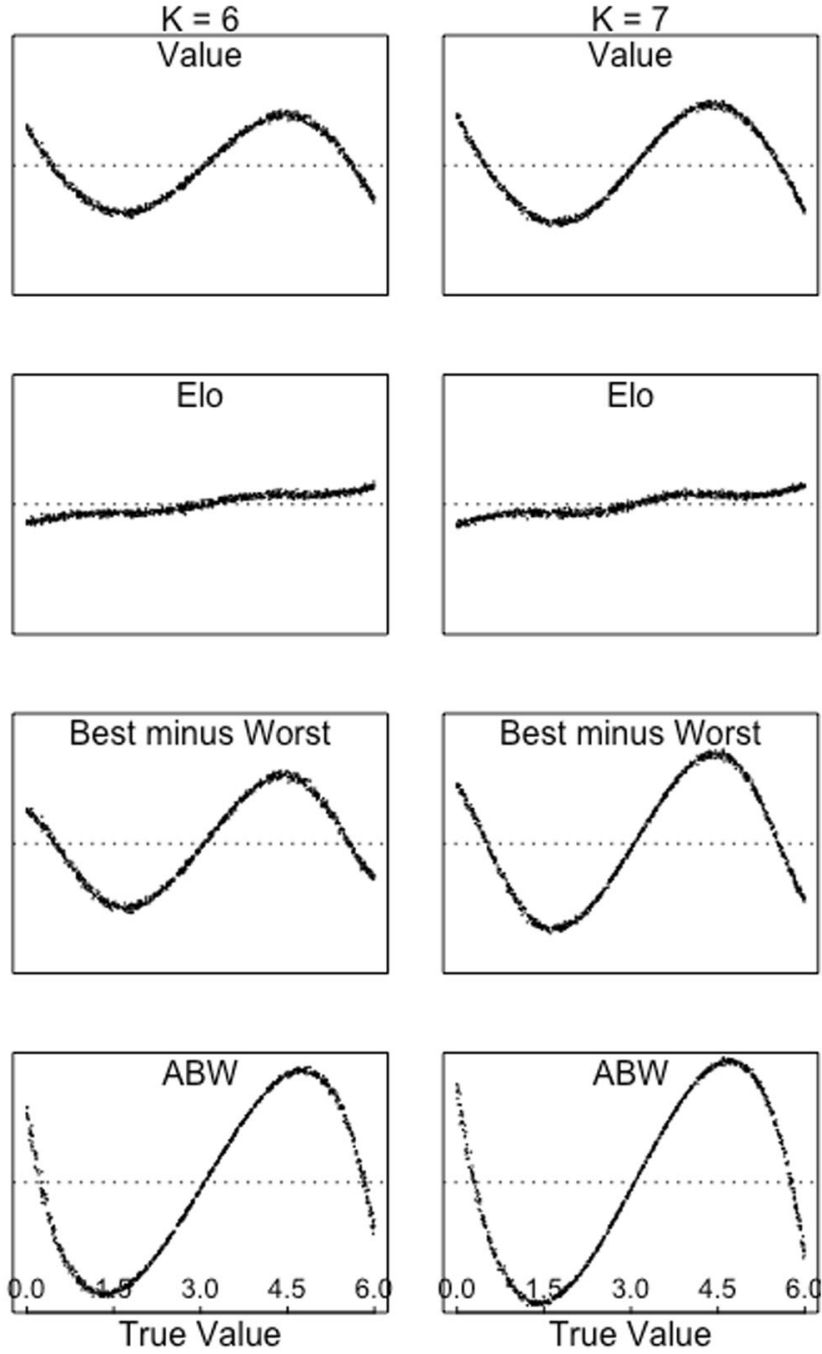

best-worst (ABW) scoring algorithms. The results are for the noise condition of 0.5 and are averaged over simulations with numbers of trials from $1 N$ to $32 N$

secondary analysis. In short, we needed to restrict our analyses in order to reduce the complexity of some figures that are presented later. The data were plotted after organizing the points into five equally sized percentile bins according to true values (1st-20th percentiles, $21 \mathrm{st}-40$ th percentiles, etc.). The results are plotted in Fig. 3.

The presented hypothesis is clearly supported for symmetric distributions. For both the normal distribution (row 1) and the uniform distribution (row 2), prediction errors are reduced at the extremes of the distribution (leftmost and rightmost bars) when large $K$ s are used, and prediction errors for items with more moderate true values (second and fourth bars) are increased. The one exception seems to be for Elo scoring, which shows reduced prediction error when $K=7$ across the entire range of true values, but we nonetheless can observe that prediction error is still minimized better for extreme than for moderate values. 

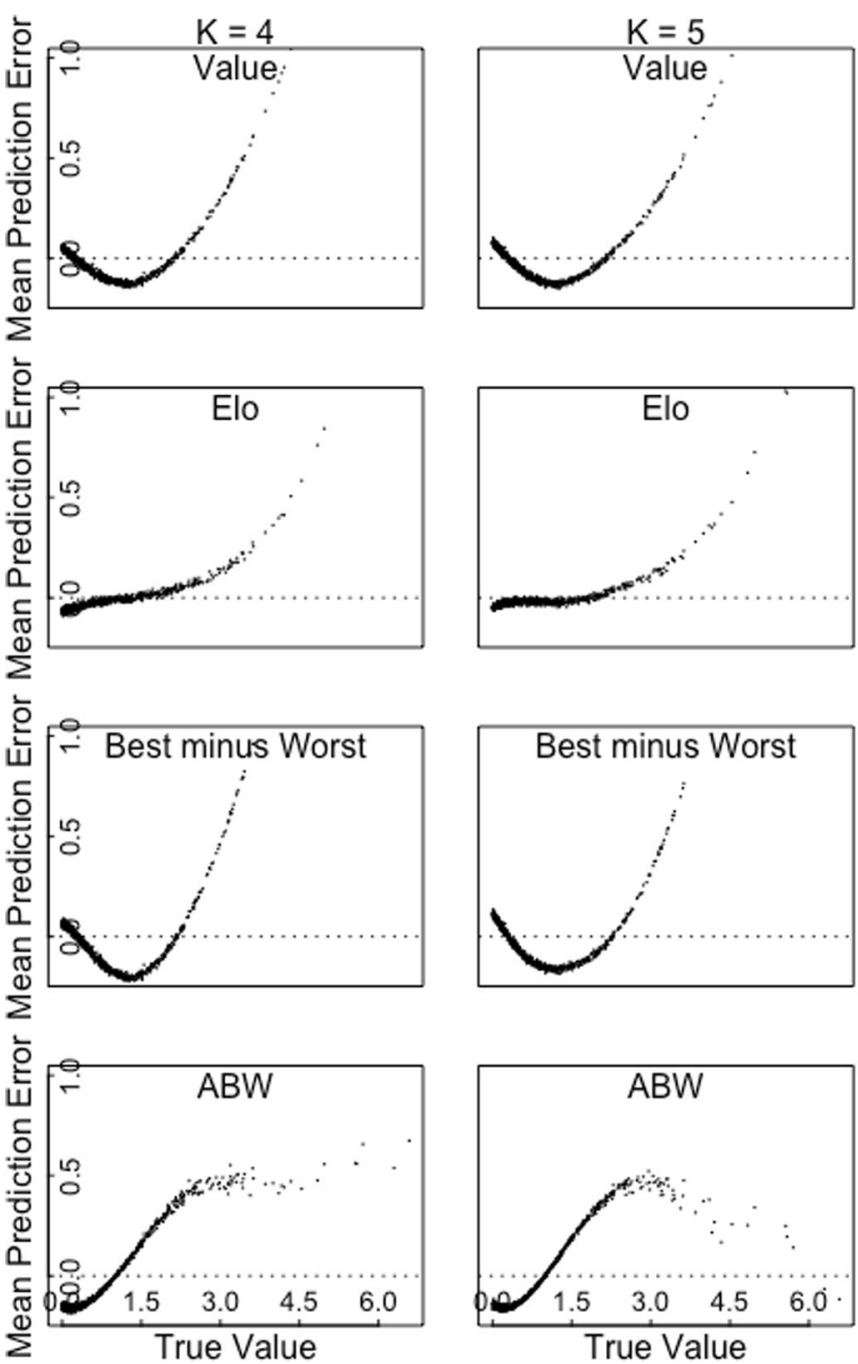

Fig. 6 Magnitudes of errors made by four scoring algorithms when scoring items with exponential decay distribution of true values. From left to right, the plot columns correspond to $K \mathrm{~s}$ of 4, 5, 6, and 7. From top to bottom, the plot rows correspond to the value, Elo, best-minus-worst,

Both of the asymmetric distributions (exponential in row 3 , $F$ in row 4 ) have a rightward skew. Thus, Bars 1 and 2 correspond roughly to the distribution's bulk, and Bars 4 and 5 correspond roughly to the distribution's tail. With this in mind, the same pattern that was seen for symmetric distributions is seen in the tails of the asymmetric distributions: Going from $K$ $=4$ to $K=7$ reduces prediction error for the most extreme values, but at the cost of inflating errors for items in the tail closest to the bulk of the distribution. The exception is bestminus-worst scoring, which shows a reduction in prediction error for all items in the distribution tails, but a greater reduction for items with more extreme true values (much like Elo scoring for symmetric distributions).

Obvious patterns are less apparent when examining the left-hand sides of the asymmetric distributions. We sidestep trying to interpret this portion of the data.

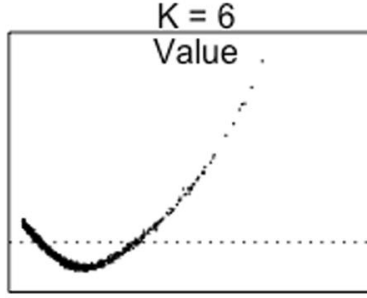

$\mathrm{K}=7$
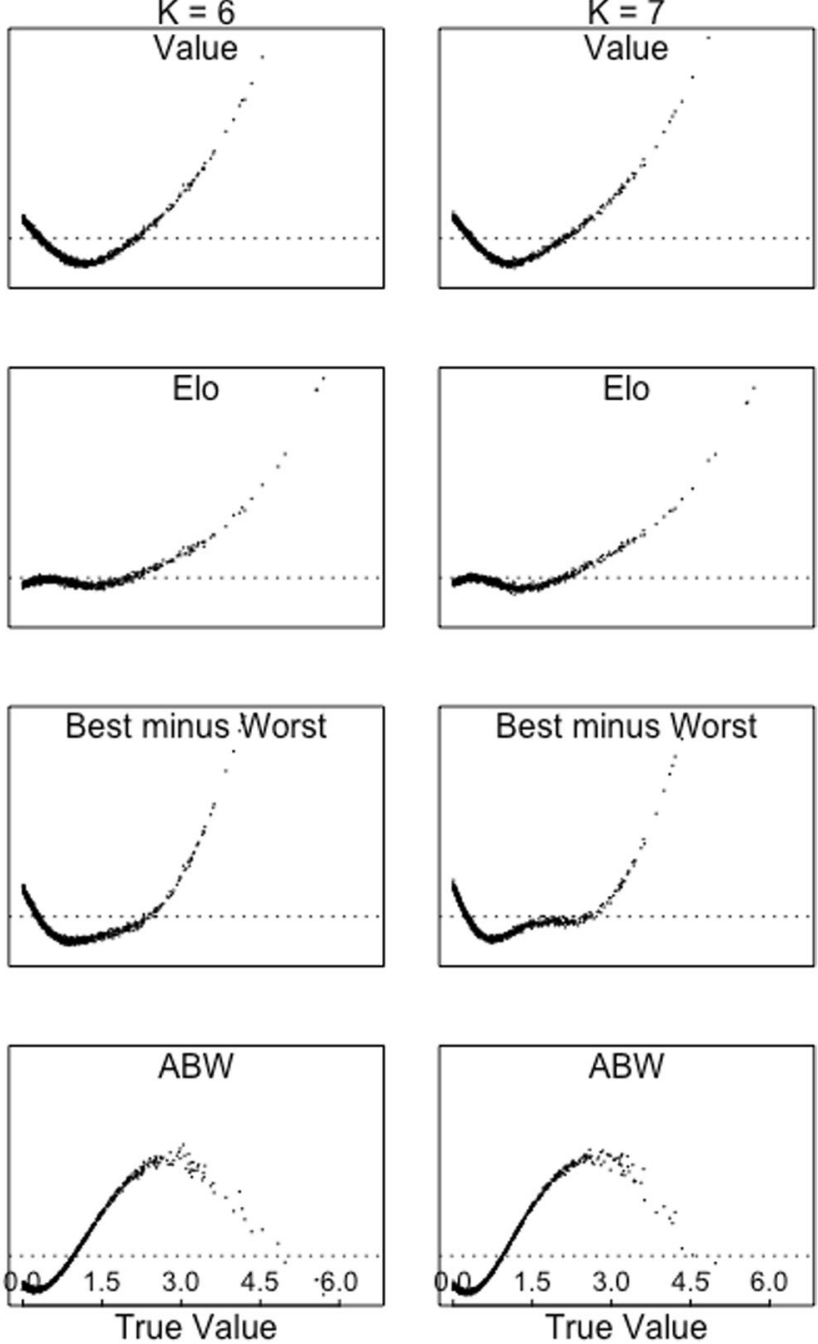

and analytic best-worst (ABW) scoring algorithms. The results are for the noise condition of 0.5 and are averaged over simulations with numbers of trials from $1 N$ to $32 N$

Figure 3 suggests that prediction errors vary by $K$, the underlying distribution of true values, and the scoring algorithm used. We now attempt to examine these prediction errors at a finer grain. For each of the 67,200 simulations run, we regressed scored values on true values for each of the six scoring algorithms and measured regression residuals. The residuals for a particular item were plotted for each level of $K$ as well as each unique true value distribution simulated. For seven levels of $K$, six different scoring algorithms, four distributions, and four levels of noise, this would result in 672 plots - too many to be interpretable. To keep results to a tractable number of figures, data are only presented for a subset of all relevant parameters. $K \mathrm{~s}$ of 2, 3, and 8 are not presented; error plots for $K=8$ were consistently similar to $K=7$. Likewise, the plots for 2 and 3 were consistently similar to that for 4 . Results are not presented for the Rescorla-Wagner 

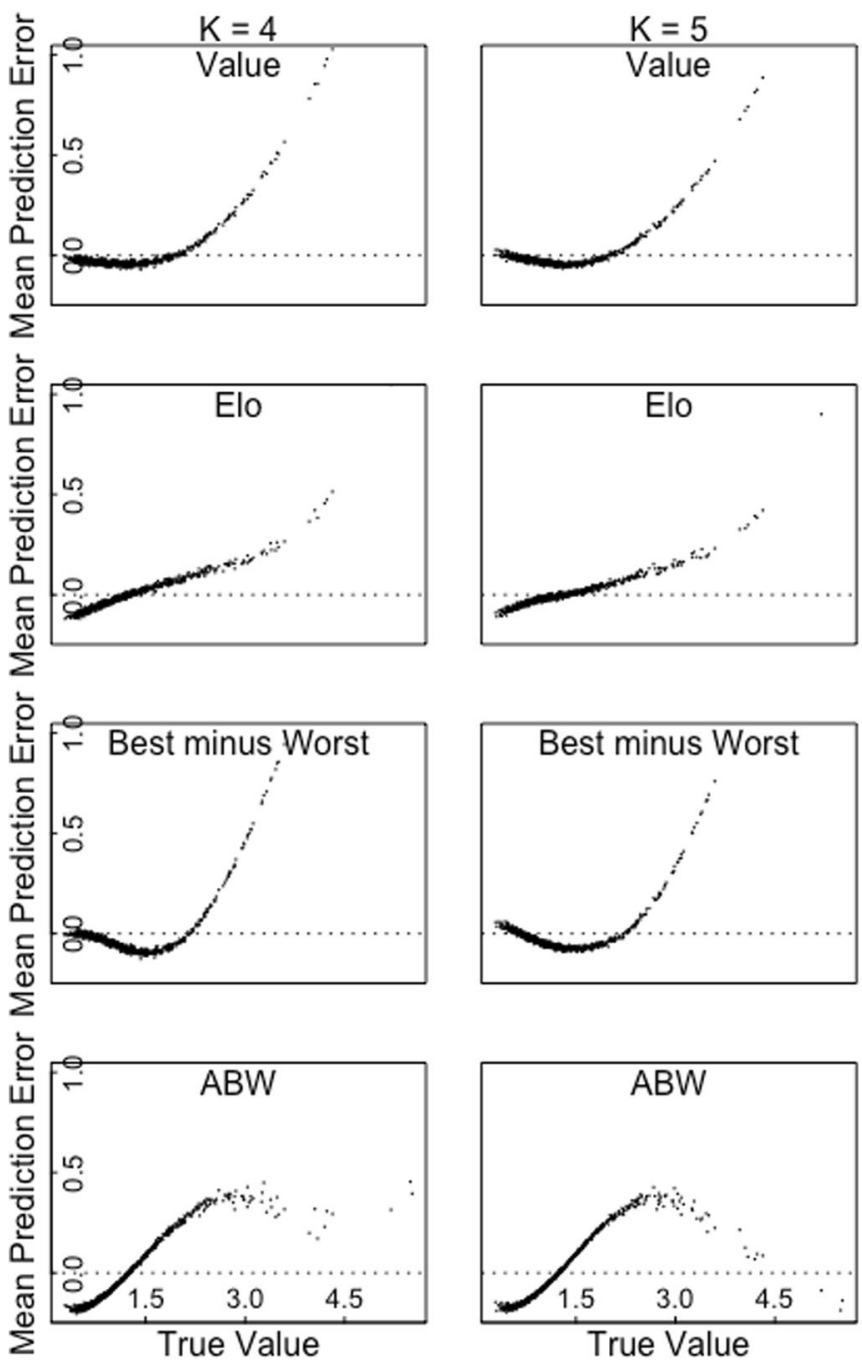

Fig. 7 Magnitudes of errors made by four scoring algorithms when scoring items whose true values have an $F(100,10)$ distribution. From left to right, the plot columns correspond to $K \mathrm{~s}$ of $4,5,6$, and 7 . From top to bottom, the plot rows correspond to the value, Elo, best-minus-worst,

scoring algorithm because its qualitative pattern of errors was consistently similar to the pattern of errors for the value scoring algorithm. Likewise, the results for the David rank scoring algorithm were similar to those for best-minus-worst scoring, so the David rank scoring algorithm is also omitted. Finally, results are only presented for noise levels of 0.5 . Under the higher noise conditions tested, the scoring algorithms all tended to converge in their patterns of prediction errors, with the caveat that Elo's prediction errors tended to be more extreme as noise levels increased, and ABW's to be less extreme. Additional figures are provided in Appendix 1, for readers who would like to compare the $0.5-S D$ noise condition, presented here, to what is observed for noise levels of $1 S D$ and 2 $S D$ s. The noiseless condition likely lacks psychological plausibility, as was discussed earlier, so it is also omitted for the sake of reducing presented figures to a manageable number.

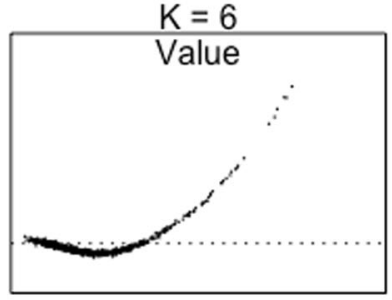

$\mathrm{K}=7$
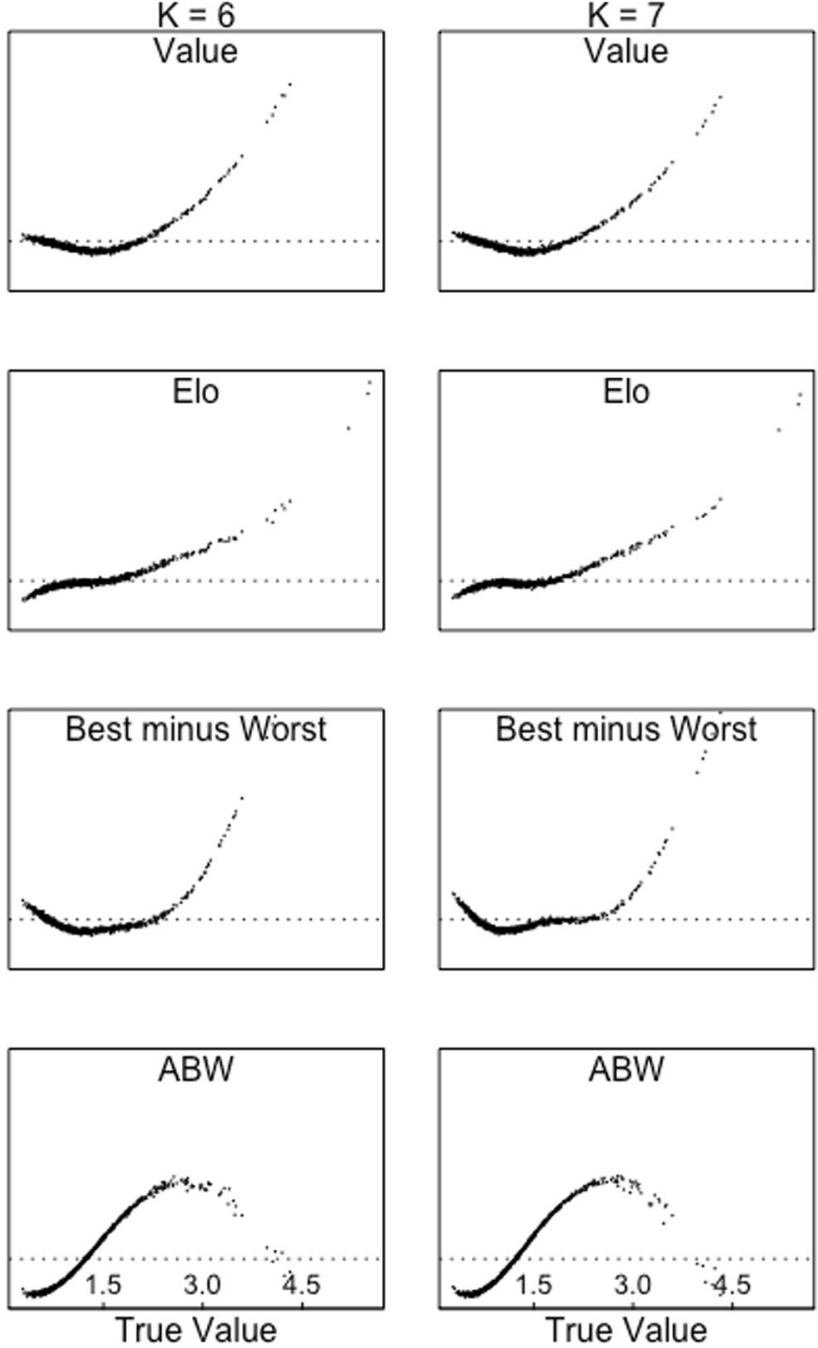

and analytic best-worst (ABW) scoring algorithms. The results are for the noise condition of 0.5 and are averaged over simulations with numbers of trials from $1 N$ to $32 N$

With these omissions considered, we now present prediction errors for four of the six scoring algorithms (value, Elo, best-minus-worst, and $\mathrm{ABW}$ ), over values of $K$ ranging from 4 to 7 , for each of the four distributions (normal, uniform, exponential, and $F$ ), using the noise level of 0.5 . The results are averaged over all numbers of trials from $1 N$ to $32 N$.

Prediction errors: Normal distribution We now present plots of prediction errors when the true values are normally distributed. The results are displayed in Fig. 4. The pattern of prediction errors across different levels of $K$ for best-minus-worst scoring (Fig. 4, row 3 ) provides a clear picture of how prediction error for extreme values is decreased as $K$ increased, but at the expense of increased prediction error for more moderate values. As Fig. 3a-d suggest, this general pattern holds true for all of the scoring algorithms except for Elo. 

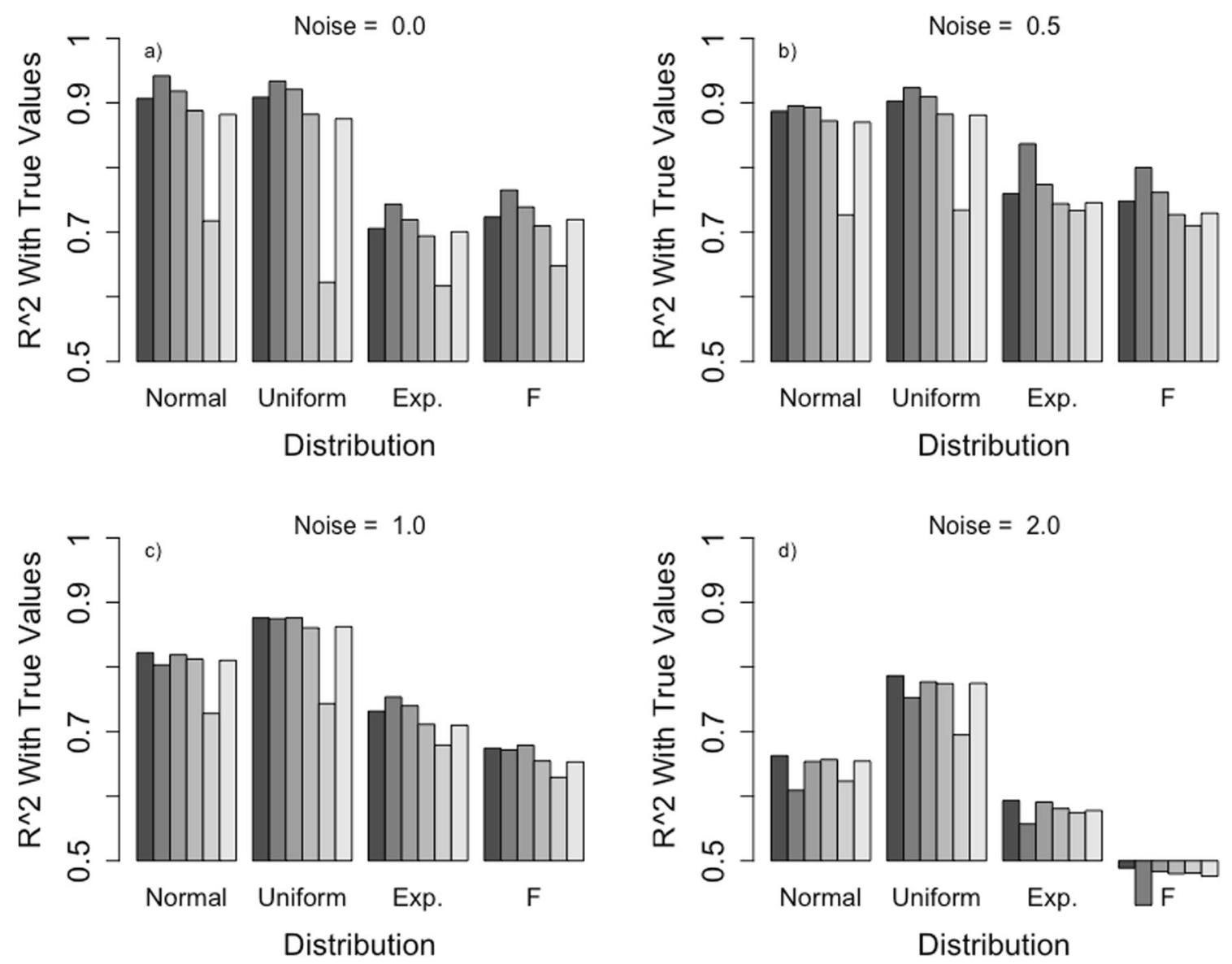

Fig. 8 Fits between derived scores and true values for different distributions, noise levels, and scoring algorithms. Data are aggregated over different levels of $K$ and different levels of simulated trials. From darkest to lightest, the bars indicate the value, Elo, Rescorla-Wagner,

best-minus-worst, analytic best-worst (ABW), and David rank order scoring algorithms. Panels a-d display the results for noise levels of $0.0,0.5,1.0$, and 2.0 , respectively

The qualitative pattern of errors produced by Elo appears relatively unaffected by $K$ under the current parameter settings. Errors made by the Elo algorithm do have one notable quality, however. For each of the three other algorithms presented, items with low-moderate true values are underestimated, whereas items with high-moderate values are overestimated. These holds across all Ks from 4 to 7 . However, Elo shows the reverse pattern for larger Ks. This difference raises a possibility: can the biases of a particular scoring algorithm be mitigated by different algorithms with contrary biases; if scores were averaged, can better estimates of true values be achieved?

Prediction errors: Uniform distribution Prediction error as a function of true value showed different patterns for uniformly distributed true values (Fig. 5) than for normally distributed true values. The prediction errors made by Elo were relatively unaffected by changes in $K$ (see also, Fig. 3f): Low values were underpredicted, and high values were overpredicted, roughly proportional to the magnitude of those values. The other three algorithms display the same sinusoidal pattern that

was characteristic of ABW for normally distributed values. Additionally, errors for each of value, best-minus-worst, and ABW scoring are magnified as $K$ increases. Also worth noting is the fact that when best-minus-worst scoring is used, the sign of the errors for the most extreme values flip as $K$ increases (Fig. 5, row 3).

Prediction errors: Exponential distribution The data for the exponential distribution are presented in Fig. 6. Unlike the previous two distributions of true values, the exponential distribution is asymmetric, with rightward skew. This appears to have consequences for prediction errors made by the algorithms. Each of the value, Elo, and best-minusworst scoring systems have clear difficulty accurately predicting values in the tail of the exponential distribution (consistently overestimating the true values). Consistent with the hypothesis about the relation between $K$ and errors for extreme values, these errors are generally reduced as $K$ increases (but not substantially; see also Fig. 3, row $3)$. Inconsistent with the prediction, errors within the bulk of the distribution are reduced (for value and best-minus- 

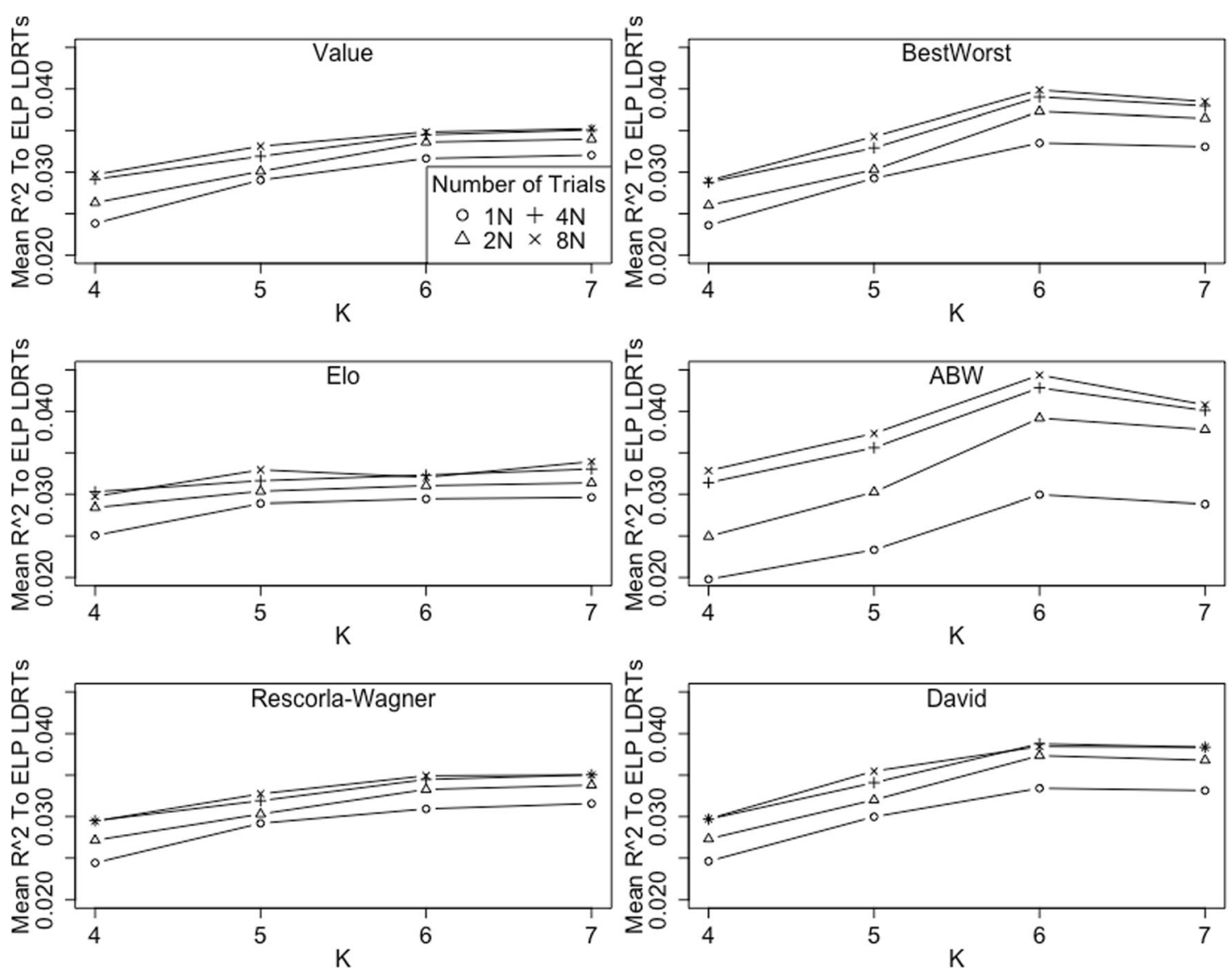

Fig. 9 Effects of $K$ and number of trials on concreteness predictive validities $\left(R^{2}\right)$ of lexical processing times (LDRTs) from the English Lexicon Project. Each panel plots the results for a different best-worst scoring algorithm. The left-hand panels present prediction-based scoring

algorithms, and the right-hand panels present counting-based scoring algorithms. Standard error bars for $1 N$ to $4 N$ trials were too small to be visible, so they are omitted

worst). Elo shows a small increase in errors for these values, consistent with predictions.

ABW stands out as having a qualitatively different pattern of prediction errors from the other three algorithms. We are unsure what to make of this difference.

Prediction errors: $\mathrm{F}$ distribution We do not discuss the patterns of errors seen for the $F$ distribution; qualitatively, they are similar to those seen for the exponential distribution. The results are displayed in Fig. 7.

Effects not dependent on K Multiple effects are observable from the presented figures that are not central to the main topic at hand (effects of $K$ ), but that bear noting for reasons of clarification or contradiction with previous suggestions in the literature. We now briefly draw attention to these secondary effects.

For all scoring methods, the more trials' worth of bestworst decisions are collected, the better the fit between the derived scores and true values. This is consistent with the simulated results of Hollis (2018) and the empirical results

of Hollis and Westbury (2018), but contrary to the recommendation of Kiritchenko and Mohammad (2017) that $2 N$ trials is sufficient to accurately estimate latent values. In all cases examined here, there is clear evidence that increasing the number of best-worst decisions progressively improves the quality of the scores produced, all other parameters being equal (see Figs. 1 and 2). The present results appear consistent with results of Hollis (2018) and Hollis and Westbury (2018), where trade-offs between measurement accuracy and data collection effort seem to be well balanced somewhere in the range of $8 \mathrm{~N}$ to $16 \mathrm{~N}$ trials.

Figure 2 gives the appearance that prediction-based scoring outperforms count-based scoring in the cases of noiseless judgments and low-noise judgments (noise $=0.5$ ), but that the reverse is true for high-noise judgments (noise $=1.0,2.0$ ). This is in fact an artifact of averaging. Although the qualitative patterns of effects pertaining to $K$ are similar within a category of scoring methods (the reason why Fig. 2 was created by averaging over scoring methods), the quantitative fits of each scoring method differ substantially. Hollis (2018) observed that the most appropriate scoring method depends on the specifics of the latent 

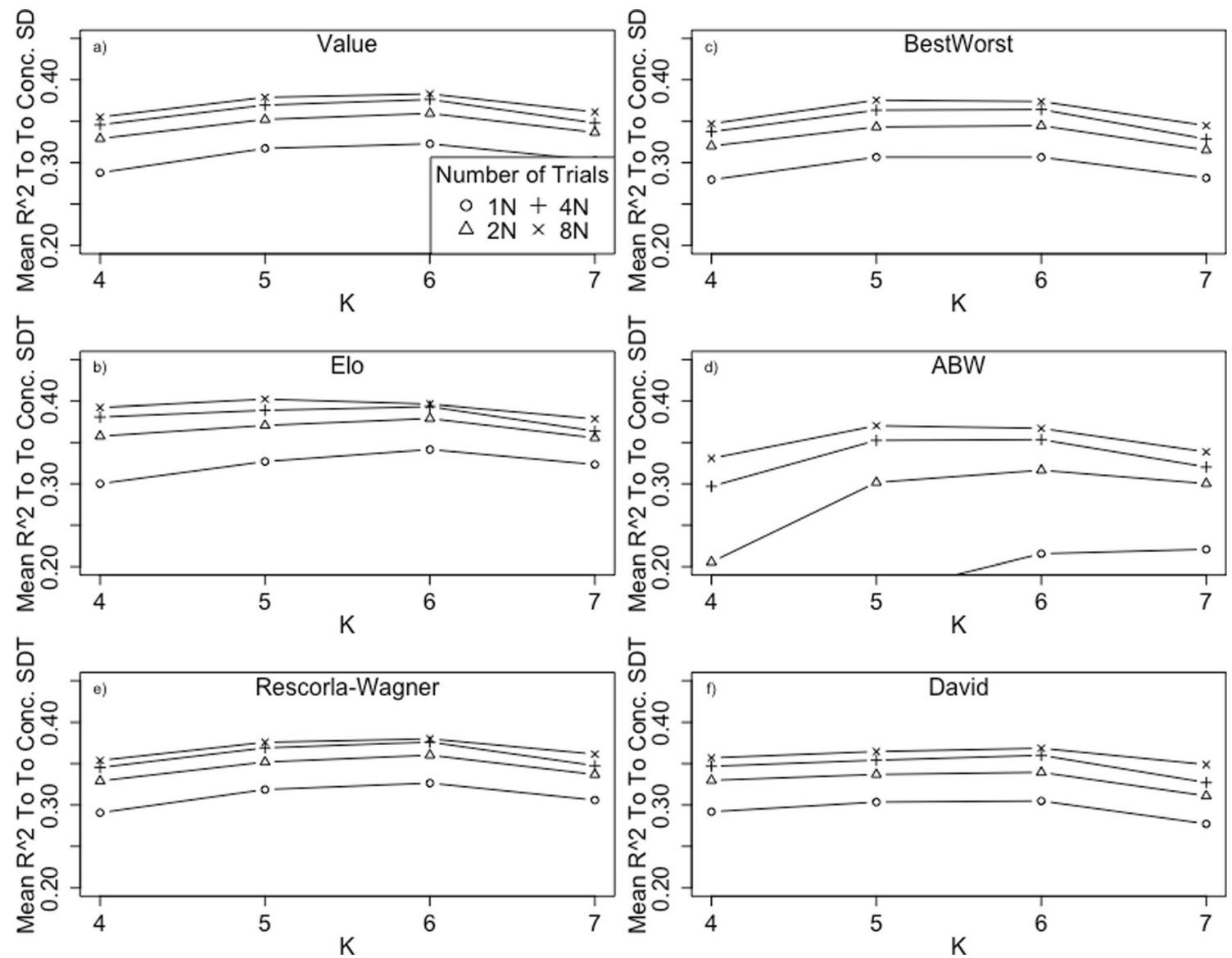

Fig. 10 Effects of $K$ and number of trials on concreteness predictive validities $\left(R^{2}\right)$ of a concrete/abstract semantic decision task. Each panel plots the results for a different best-worst scoring algorithm. The left-

hand panels present prediction-based scoring algorithms, and the righthand panels present counting-based scoring algorithms. Standard error bars for $1 N$ to $4 N$ trials were too small to be visible, so they are omitted

value's underlying distribution and the amount of noise in the judgments. Figure 8 presents fits to true values, broken down by distribution, noise level, and scoring algorithm (and averaged over $K$ and number of trials).

Although prediction-based methods on average perform better than count methods for noise levels of 0.0 and 0.5 , it is clear that this effect is mostly being driven by exceptionally good fits for Elo and exceptionally poor fits for ABW. As noise levels get higher, Elo begins performing relatively poorly as compared to the other scoring algorithms and $\mathrm{ABW}$ begins performing relatively well. The other four scoring algorithms display much less variation when compared amongst each other, on average, across the various simulation parameters tested.

The results of Hollis (2018) suggested that value scoring consistently produces the highest quality scores for noise levels of 1.0 and 2.0, regardless of distribution. That is not the case in the present data. It is worth noting that Hollis (2018) only simulated the case of $K=4$, whereas the data from Fig. 8 are averaged over $K$ s ranging from 2 through 8 .

We reiterate a recommendation offered by Hollis (2018): Since all scoring algorithms can be calculated over the same data, there is no strong reason to prefer one over others a

priori. All should be computed, their scores should be validated against an appropriate measure (e.g., lexical processing times, sentiment classification hit rate, etc.), and the most predictive set of scores for that particular dataset should be used. Software implementing all six of the discussed scoring algorithms is available at www.ualberta.ca/ hollis .

\section{Discussion}

The main purpose of this experiment was to systematically explore the role of the number of items per trial, $K$, affecting the quality of best-worst judgments in large- $N$ experiments. This is a parameter of best-worst scaling that has not been rigorously studied, and there is reason to doubt that the convention of $K=4$ is optimal in the general case.

The presented work provides consistent evidence that 4 is rarely the preferred value for $K$. Depending on the amount of noise in the data, the number of trials data are being collected for, and the scoring algorithm being used, $K$ s ranging from 3 to 8 may all produce scores that are more strongly correlated with true values. Unfortunately, this means there is no straightforward answer for how to best choose $K$. Unfortunately, unlike number 

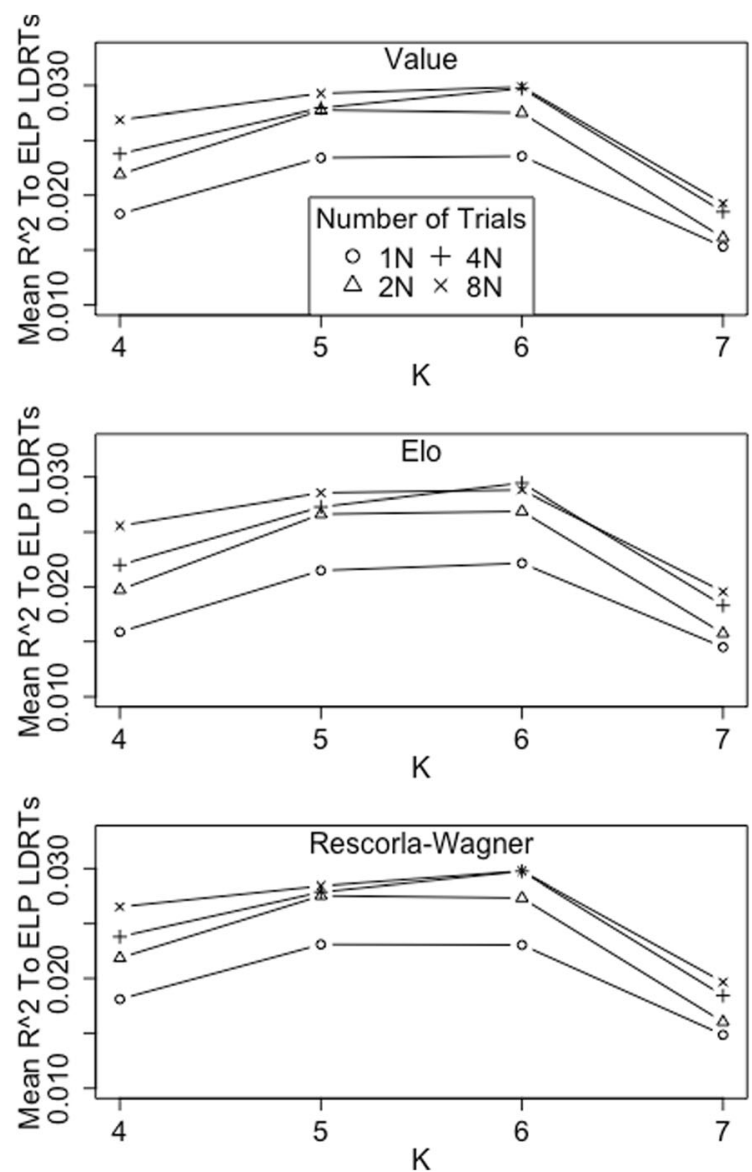

Fig. 11 Effects of $K$ and number of trials on word arousal predictive validities $\left(R^{2}\right)$ of lexical processing times (LDRTs) from the English Lexicon Project. Each panel plots the results for a different best-worst scoring algorithm. The left-hand panels present prediction-based scoring algorithms, and the right-hand panels present counting-based scoring

of trials and scoring algorithm, a final decision about $K$ must be made before data collection begins.

As a first step to choosing an appropriate value of $K$, researchers may want to start by asking "do I expect the data that will be produced to be particularly noisy?"; if the answer is "yes," a larger $K$, probably at least 8 , should be preferred (see Fig. 2c, d, g, and h). This will likely mean that Elo and ABW scoring will be poor scoring methods, but all of the others should be roughly equivalent. This advice is likely incomplete, in that it ignores the fact that the present simulations did not build in a mechanism to account for the increased cognitive load that comes with having to make judgments over more items. This point will be addressed in a later experiment.

Best-worst scaling has been demonstrated to be able to not just infer rank information, but also information about relative spacing along the underlying latent dimension. The ability to reconstruct spacing information is dependent on noisy judgment (Hollis, 2018): Uncertainty about rank gives information about relative distance. Such information is not available in noiseless judgments. When noise levels are very high and $K$ is
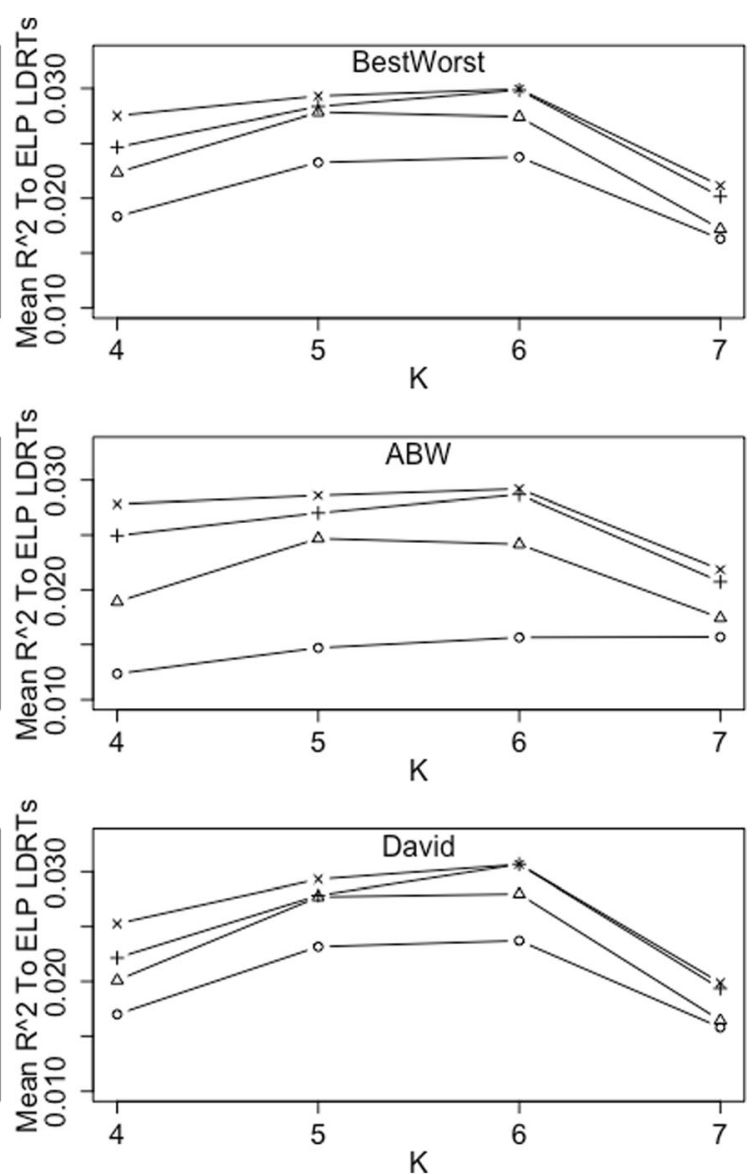

algorithms. Data for $1 N$ to $4 N$ trials were simulated by subsampling from the full $8 N$ trials collected. In all, 100 such subsamplings were conducted for each combination of $K$ and trial number. Standard error bars were small enough that they interfered with viewing the symbols used for the different numbers of trials, so they are omitted

very small, the likelihood that the best and worst items will be confused is increased, as compared to when $K$ is higher.

We suspect that employing large $K \mathrm{~s}$ in high-noise conditions will allow for more discrimination about relative rank than if smaller $K$ s were used. When using large but not smaller $K \mathrm{~s}$, a best (or worst) item will be confusable with some of the $K$ items present in this trial, but not all of them. This is simply because items with a wider range across the distribution of true values will be sampled on any given trial.

When the decision that participants need to make is anticipated to have low noise, smaller Ks should be preferred. This is particularly clear for the extreme case of no noise and countbased scoring algorithms (Fig. 1d-f). Prediction-based scoring algorithms are notably more robust to $K$ when data are noiseless (Fig. 1a-c). When noise levels that more realistically reflect psychological decision making (e.g., noise value of 0.5 ; see Fig. $2 \mathrm{~b}$ vs. $2 \mathrm{f}$ ) are present, the ideal $K$ is in the 6-8 range, depending on the number of trials for which data are being collected. Hollis and Westbury (2018) have previously suggested that $8 N$ trials balances the cost of data collection with 

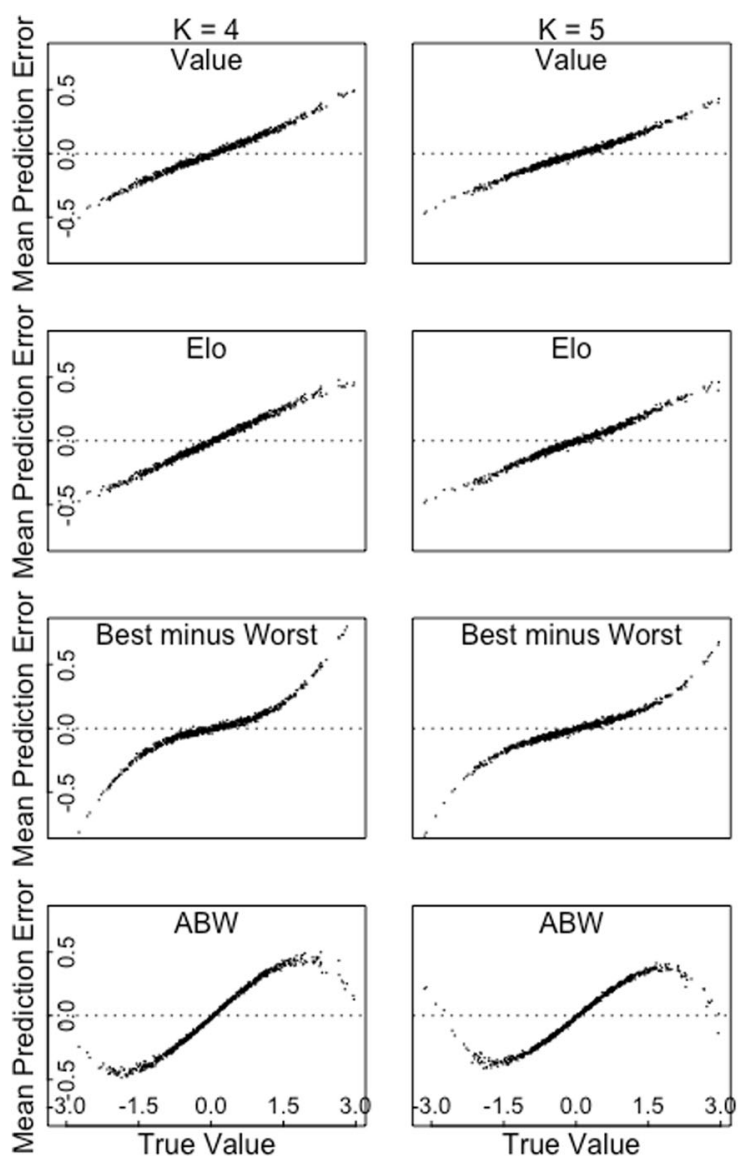

Fig. 12 Magnitudes of errors made by four scoring algorithms when scoring items with normally distributed true values. From left to right, the plot columns correspond to $K$ s of 4, 5, 6, and 7. From top to bottom, the plot rows correspond to the value, Elo, best-minus-worst, and analytic

the accuracy of derived scores fairly well. At this number of trials, researchers may want to consider $K=6$ for count-based scoring methods, and $K=8$ for prediction-based scoring methods. Of course, to reiterate a point made multiple times already, there is no strong reason to precommit to using only one scoring algorithm. All can be calculated on the same data, and the best-fitting one can be chosen as long as reasonable steps are taken to validate the fits and to avoid overfitting. With this in mind, a $K$ of 6 is the suggested value for giving each scoring algorithm its best opportunity to fit the data well.

It is important to give each scoring algorithm its best opportunity to fit the data well. The places along the latent dimension where each scoring algorithm makes its largest and smallest errors vary substantially by algorithm, $K$, and the distribution of true values. This is, again, another reason why researchers should not precommit to using only one scoring algorithm. All should be used, fit to the relevant data, and compared on their errors to choose the scoring method that is going to be most useful for the needs at hand. If the highest priority is to minimize errors in the extreme cases, a researcher might wish to go with one scoring algorithm over another even if the other better predicts the entire range of values. Figures 4, 5, 6, and 7 provide
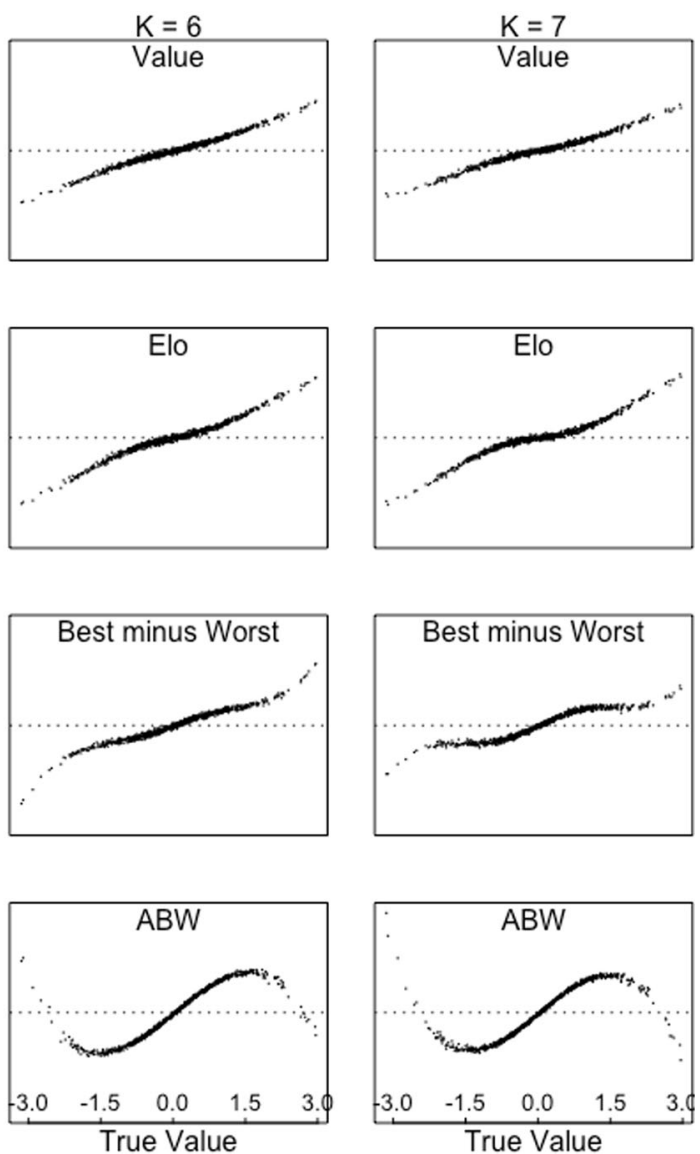

best-worst (ABW) scoring algorithms. Results are shown for the noise condition of 1.0 and are averaged over simulations with numbers of trials from $1 N$ to $32 N$

visual guides for choosing the $K$ that will best minimize errors at certain places along the range of possible true values for each of the six scoring algorithms presented, given simple assumptions about the distribution of true values.

In conclusion, a $K$ of 4 appears far from ideal for large- $N$ best-worst scaling experiments. Larger values of $K$ are preferred. In the general case in which minimal assumptions can be made about data noisiness or distribution of true values, we recommend a $K$ of $6,8 N$ trials, and testing and validating all scoring algorithms on the basis of these presented results taken as a whole. If researchers can make stronger assumptions about the noise conditions and the distribution of true values, different values of $K$ may be preferable.

We have previously identified three sources of influence on $K$ 's role in data quality. One, as the number of items per trial increases, so does the absolute amount of rank information gathered. This creates a gradient toward larger $K$ s producing more informative data. However, as $K$ increases, that information becomes proportionally more about relationships to extreme values. We anticipated that this would mean that very high-value $K$ s would increase measurement accuracy for extreme values at the expense of accuracy for less-extreme values, 

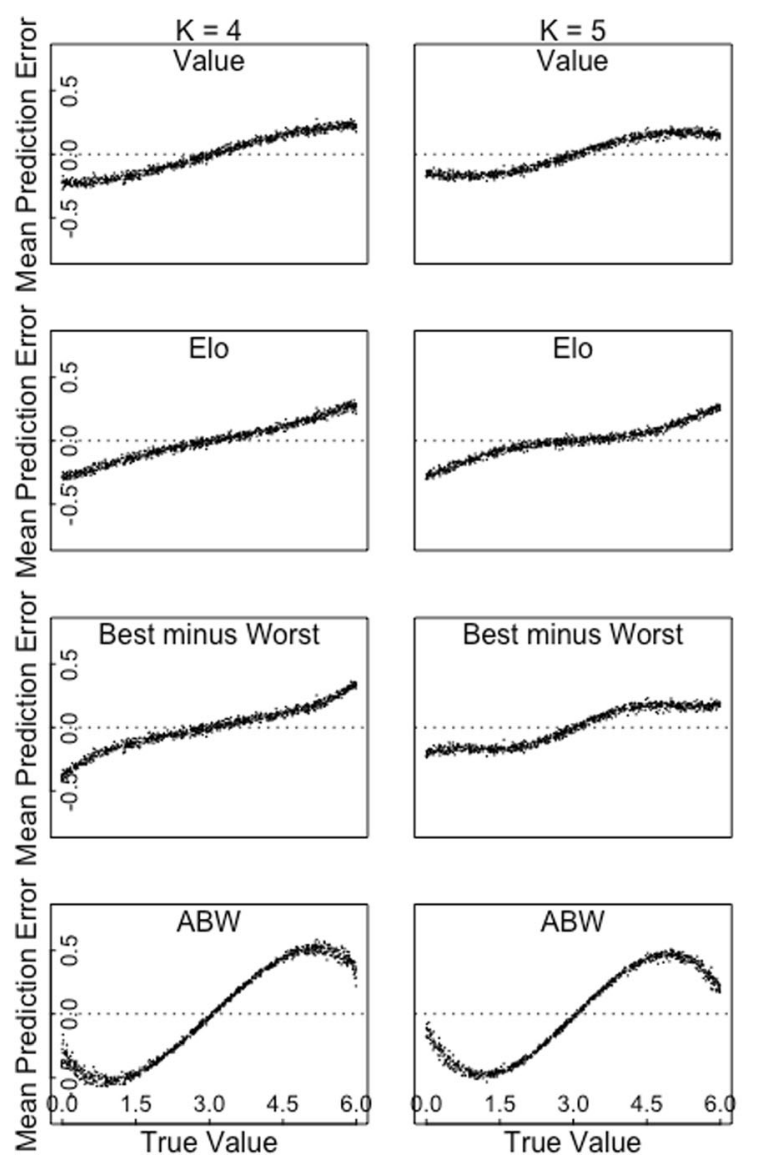

Fig. 13 Magnitudes of errors made by four scoring algorithms when scoring items with uniformly distributed true values. From left to right, the plot columns correspond to $K \mathrm{~s}$ of $4,5,6$, and 7 . From top to bottom, the plot rows correspond to the value, Elo, best-minus-worst, and analytic

in turn creating a gradient to prefer smaller $K$ s. These two opposing forces should create a situation such that data quality is increased as $K$ increases up to a threshold, and then the data quality should decrease. Although Fig. 3 suggests that our predictions about how measurement error will be in a trade-off with $K$ are oversimplified (particularly for asymmetric distributions), the data still support the general conclusions that there is a threshold beyond which increasing $K$ decreases the data quality, but that threshold is larger than $K=4$ in most cases.

These simulations have neglected a third source of influence on data quality as a function of $K$ : Increasing $K$ also increases the cognitive demands on participants, which is likely to introduce more errors. This may, in turn, increase or decrease the predictive validity of best-worst data. To test how this cognitive component of best-worst scaling might affect data, we now turn to a behavioral study.

\section{Experiment 2}

The primary purpose of Experiment 2 was to test whether the simulation results of Experiment 1 accurately model results from
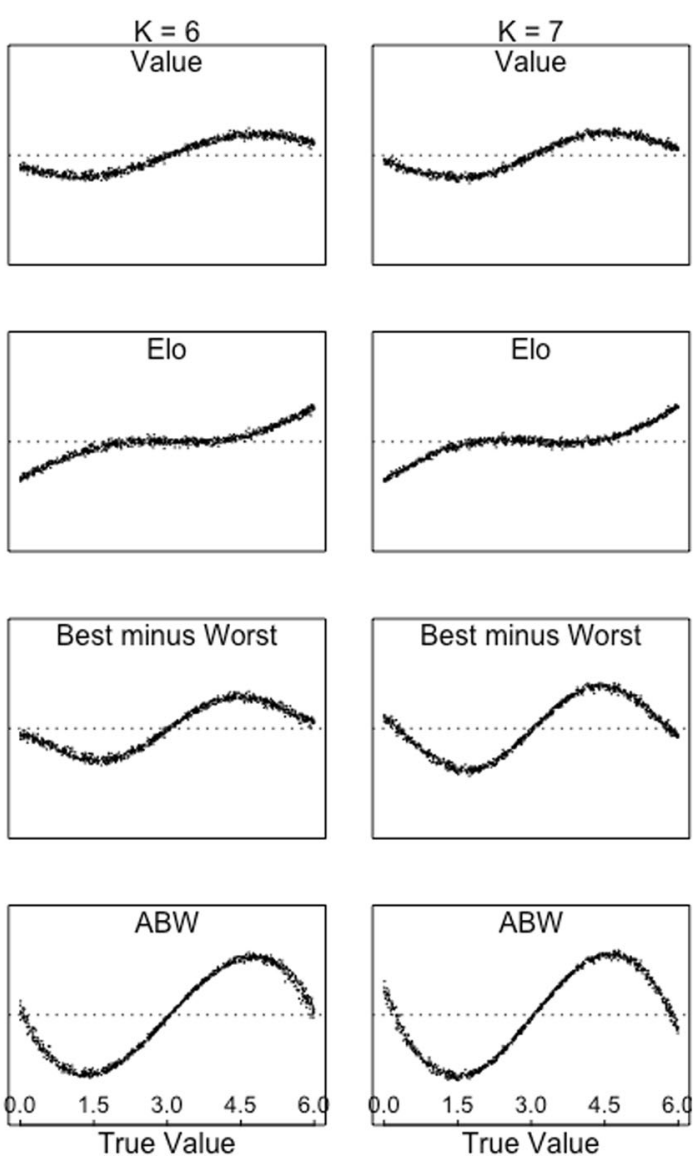

best-worst $(\mathrm{ABW})$ scoring algorithms. Results are shown for the noise condition of 1.0 and are averaged over simulations with numbers of trials from $1 N$ to $32 N$

behavioral studies when $K$ was manipulated. The central hypotheses were that $K$ and data quality should have an invertedU-shaped relationship, and that increasing $K$ should more clearly be a detriment in the behavioral case than in the simulation case. This second prediction is due to noise being introduced by the increased cognitive load of trying to decide on the best and worst items over incrementally larger list sizes. This hypothesized cognitive load component was not modeled in Experiment 1.

Most large- $N$ applications of best-worst scaling have involved measuring the semantic properties of words. Because of this currently narrow scope of the use of large- $N$ best-worst studies, the following behavioral study was designed to be complementary to the already-existing literature on norm collection and best-worst scaling.

\section{Method}

Items Data were collected for a total of 420 items, sampled randomly from the intersection of the Affective Norms for the English Language (ANEW; Bradley \& Lang, 1999), the English Lexicon Project (Balota et al., 2007), the British Lexicon Project (Keuleers, Lacey, Rastle, \& Brysbaert, 

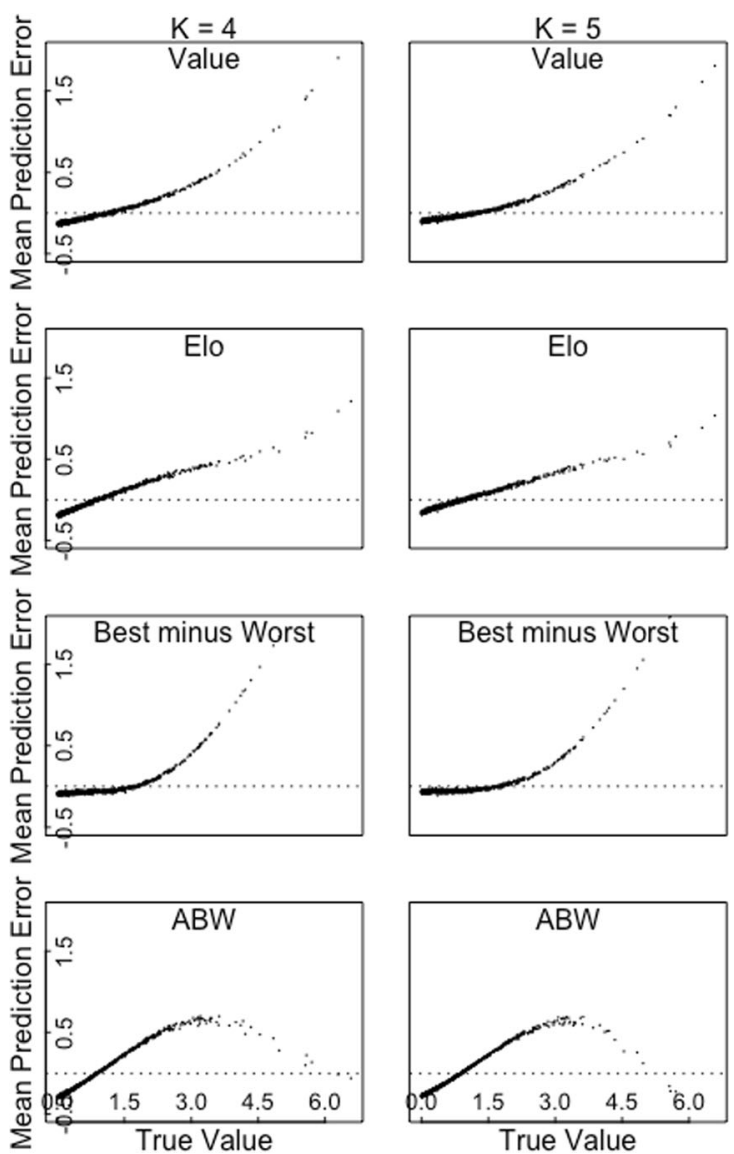

Fig. 14 Magnitudes of errors made by four scoring algorithms when scoring items with exponentially distributed true values. From left to right, the plot columns correspond to $K \mathrm{~s}$ of 4,5 , 6, and 7. From top to bottom, the plot rows correspond to the value, Elo, best-minus-worst, and

2012), and Brysbaert's concreteness norms (Brysbaert, Warriner, \& Kuperman, 2014). A total of 420 items were used because this number ensured that within each value of $K$ tested, all items could occur equal numbers of items, and that between values of $K$, data could be collected for equal numbers of trials. This design allowed for the most direct comparison of the effects of $K$ and number of trials.

Values of K Data were collected for $K$ s of 4, 5, 6, and 7.

Number of trials In all, $8 N(420 * 8=3,360)$ trials worth of data were collected for each value of $K$. The trials were constructed such that each item appeared an equal number of times, and no item pair occurred more than once.

Latent dimensions Participants were asked to make either a concreteness judgment (does this correspond to a concept detectable by the senses?) or an arousal judgment (is this concept activating or inhibiting?) about the presented items. These two dimensions were chosen on the basis of their relative noisiness. Word concreteness judgments are known to be highly reliable (e.g., Brysbaert et al., 2014), whereas there is fair
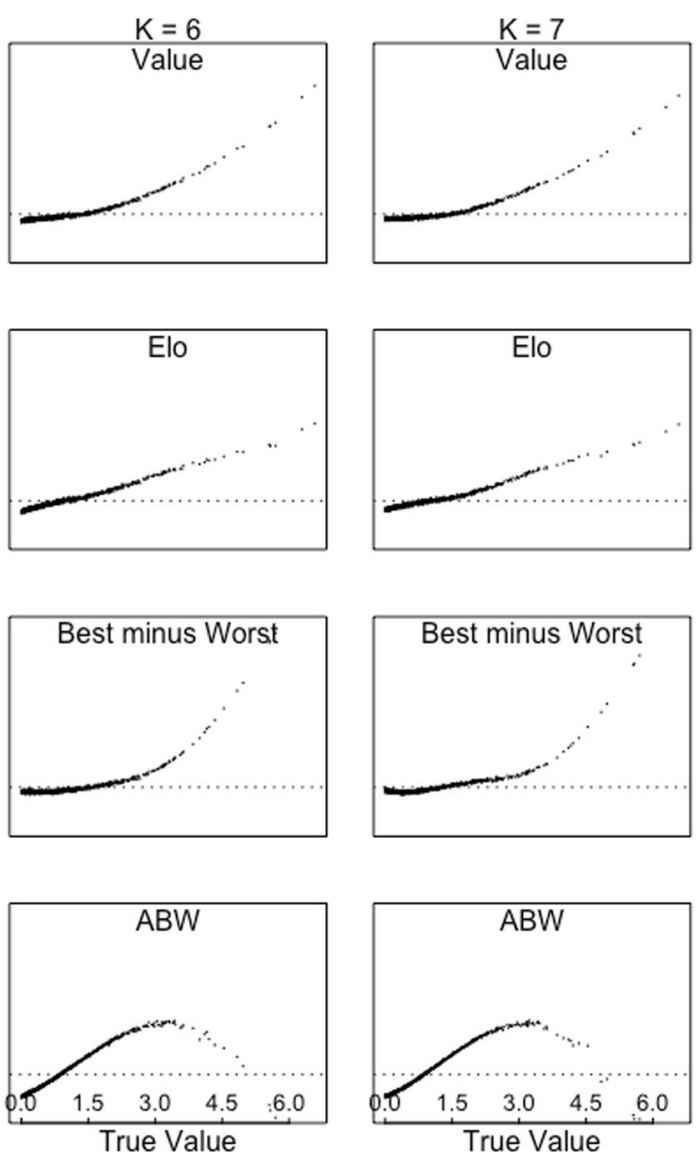

analytic best-worst $(\mathrm{ABW})$ scoring algorithms. Results are shown for the noise condition of 1.0 and are averaged over simulations with numbers of trials from $1 N$ to $32 N$

evidence that arousal judgments are comparably noisy (e.g., Hollis \& Westbury, 2018; Hollis, Westbury, \& Lefsrud, 2017; Westbury et al., 2013). Hollis and Westbury (2018) collected best-worst norms for five semantic dimensions: valence, arousal, subjective frequency, age of acquisition, and concreteness. They found that concreteness scores were the most discriminable of the five dimensions, whereas arousal scores were least discriminable. This suggests that these two dimensions are good candidates for "low-noise" and "high-noise" conditions of judgment, which is why they were chosen. The participant instructions were taken, with minimal adaption, from Appendix 1 of Hollis and Westbury (2018).

Procedure Participants were presented with a set of $K$ items and instructed to choose the best and worst items in that set on some specified underlying latent dimension. Participants would complete at least one list containing 20 trials, but had the option of completing up to four lists for a specific value of $K$. Each value of $K$ was run as a separate experiment and, consequently, a person could in theory contribute responses to 2 (judgment conditions) $\times 4$ (values of $K$ ) $\times 4$ (maximum number of lists $) \times 20$ (trials per list) $=640$ trials. After all data 

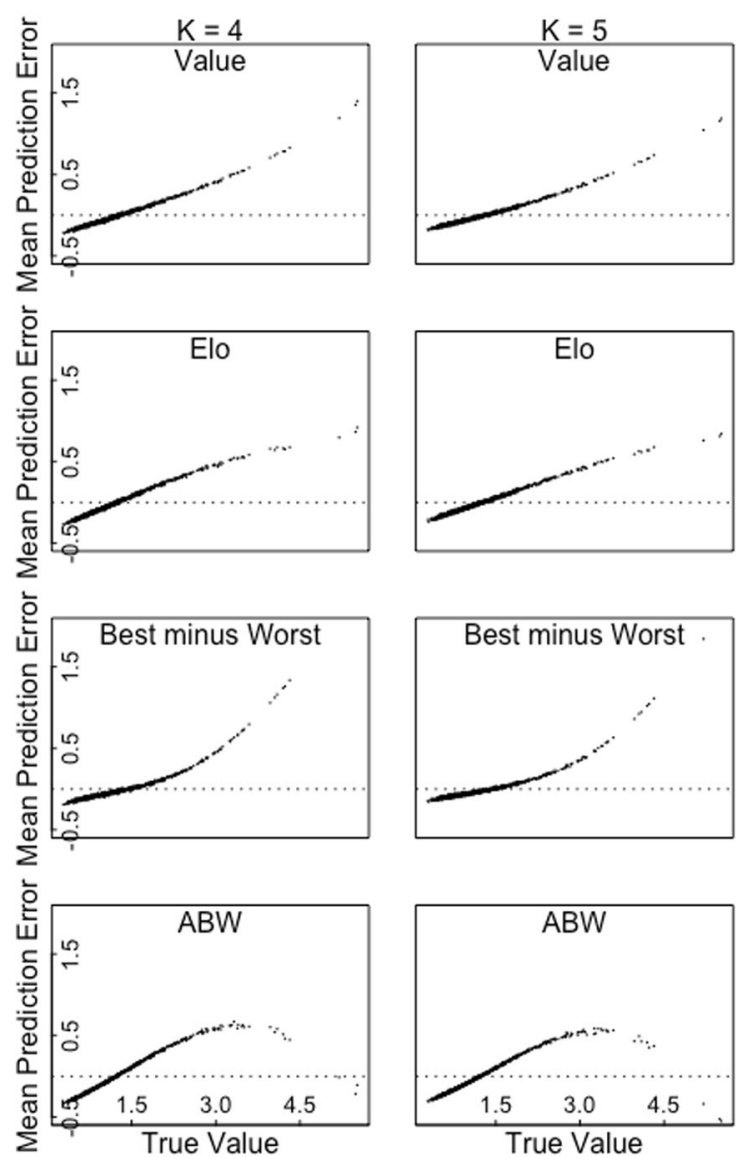

Fig. 15 Magnitude of errors made by four scoring algorithms when scoring items with $F$-distributed true values. From left to right, the plot columns correspond to $K \mathrm{~s}$ of 4, 5, 6, and 7. From top to bottom, the plot rows correspond to the value, Elo, best-minus-worst, and analytic best-

were collected, they were scored according to each of the six scoring algorithms discussed above. In lieu of knowledge about the true values, scores were validated against behavioral measures of lexical processing.

Participants All data were collected from the CrowdFlower crowdsourcing platform. Participants were restricted to English-speaking persons who resided in Canada or the United States. Age and gender information was not collected. For arousal judgments with $K=4,5,6$, and 7, totals of 68, 64, 66 , and 66 unique participants contributed judgments. For concreteness judgments, $66,67,65$, and 66 unique participants contributed judgments. Overall, the average contribution was 50.9 trials per participant per judgment condition. Each judgment condition consisted of 3,360 trials. Most participants contributed to multiple judgment conditions. A total of 151 unique participants contributed data across all judgment conditions.

Validation criterion We used two validation criteria for evaluating the quality of scores derived from best-worst scaling data: lexical decision times from the English Lexicon Project
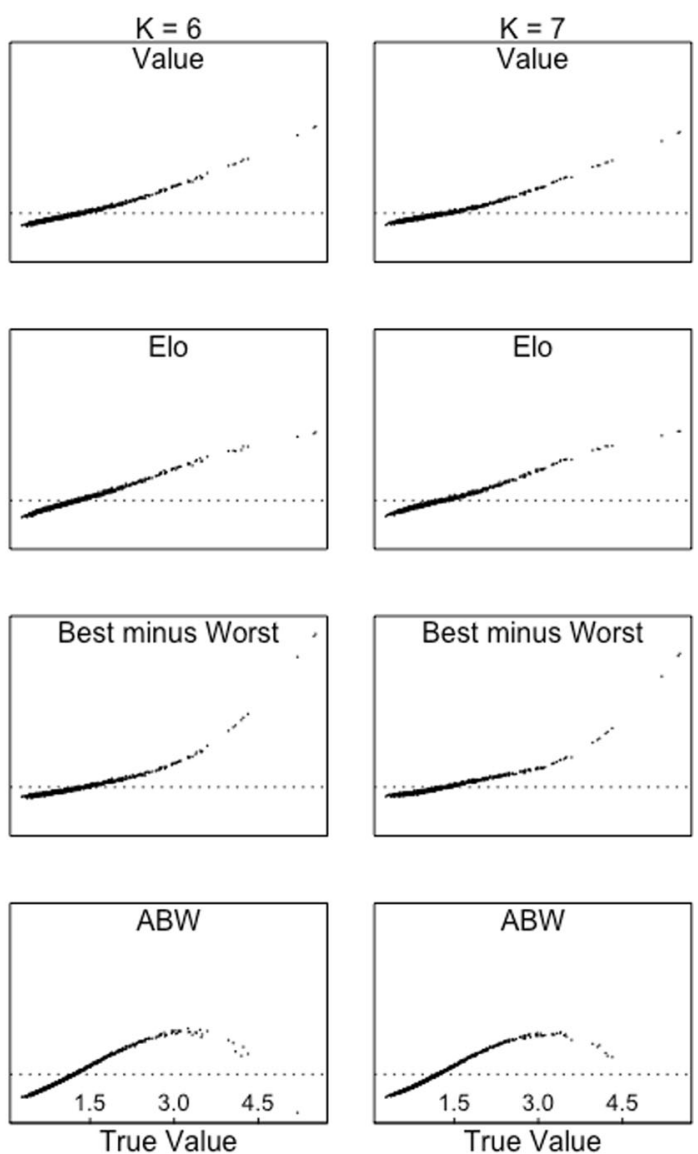

worst (ABW) scoring algorithms. Results are shown for the noise condition of 1.0 and are averaged over simulations with numbers of trials from $1 N$ to $32 N$

(Balota et al., 2007), and concreteness decision times from Pexman, Heard, Lloyd, and Yap (2017). Both of these measures tap into cognitive processes of recognizing words and their meanings. Insofar as a semantic property contributes to lexical processing, better estimates of that semantic property should more strongly correlate with measures of lexical processing. Both concreteness (Paivio, 2013) and arousal (Kuperman et al., 2014) have been implicated in lexical processing.

Further information We refer readers to Hollis and Westbury (2018) for more detailed information about how the trials were constructed and how noncompliant participants were identified and filtered from the experiment. The present experiment followed the same methodology used in that previous work.

\section{Results}

Estimating word concreteness Results for concreteness decisions are presented first.

A subsampling procedure was used to simulate the results for cases in which data are only collected for $1 N, 2 N$, and $4 N$ 

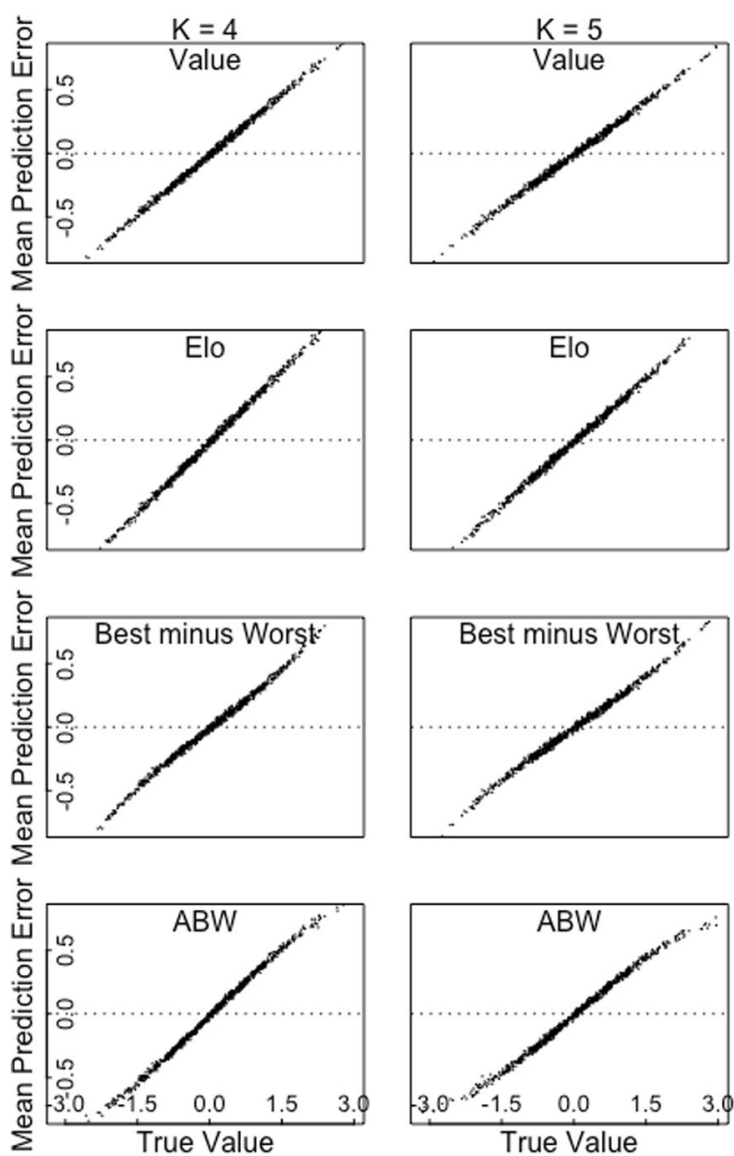

Fig. 16 Magnitudes of errors made by four scoring algorithms when scoring items with normally distributed true values. From left to right, the plot columns correspond to $K \mathrm{~s}$ of 4, 5, 6, and 7. From top to bottom, the plot rows correspond to the value, Elo, best-minus-worst, and analytic

trials (vs. $8 \mathrm{~N}$ in this experiment). Trials for a particular value of $K$ were partitioned into subsets so that each item appeared exactly once in each subset. Subsets were then sampled randomly without replacement, to construct lists of $1 \mathrm{~N}, 2 \mathrm{~N}$, or $4 \mathrm{~N}$ trials. The subsampling procedure was repeated 100 times for each pair of $K$ and the number of trials. This procedure for estimating the effects of fewer numbers of trials was identical to that used by Hollis and Westbury (2018).

Figure 9 presents the effects of $K$ and number of trials on the predictive validity of lexical decision response times taken from the English Lexicon Project (Balota et al., 2007).

The main results pertaining to $K$ are largely consistent with the simulations from Experiment 1: (a) Increasing $K$ beyond 4 increases the fit of the best-worst scores to the validation measure (in this case, lexical processing times from the English Lexicon Project), (b) count-based scoring methods show a decrement in predictive validity past a critical value of $K$ (in this case, 6 ), and (c) prediction-based scoring methods show more robustness to choices of $K$ when $K$ is large than do count-based scoring methods.

Of additional note is the fact that count-based scores of concreteness better fit the ELP processing times than
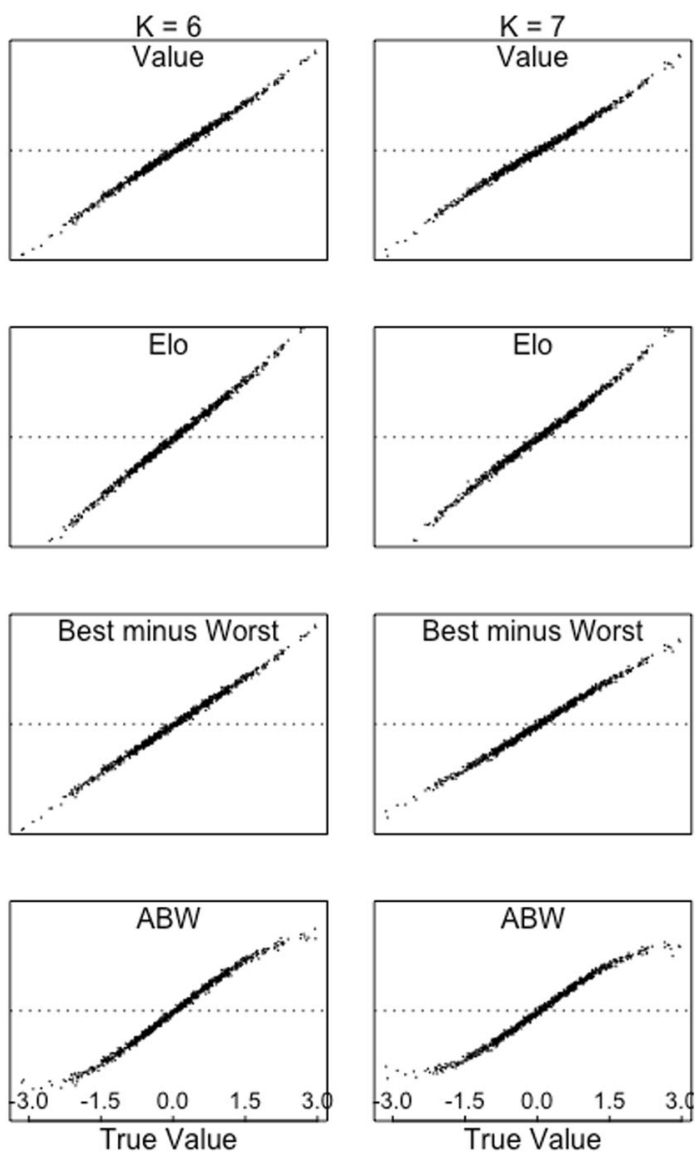

best-worst (ABW) scoring algorithms. Results are shown for the noise condition of 2.0 and are averaged over simulations with numbers of trials from $1 N$ to $32 N$

do prediction-based scoring methods, with $\mathrm{ABW}$ standing out as the best-fitting scoring algorithm. This is consistent with the results observed by Hollis and Westbury (2018). However, we remind readers that the error profiles generated by these scoring algorithms can be quite different (e.g., Figs. 4, 5, 6, and 7), and it may be worth examining the error profiles before committing to one scoring algorithm over another. We omit such an analysis here, to maintain a focus on the central research question at hand: the effects of $K$.

These analyses were repeated for the Pexman concreteness semantic decision time (SDT) data (Pexman et al., 2017), in which participants were shown high- and lowconcreteness words and instructed to make a decision about that concreteness status as quickly as possible. The concreteness SDT data had low coverage with our norms $(n=154)$, since our norms were sampled continuously across the range of concreteness values, but the Pexman et al. stimuli were sampled to be items with extreme concreteness values. Nonetheless, the analysis reproduced two of the three key patterns seen with the ELP results. Data are presented in Fig. 10. 

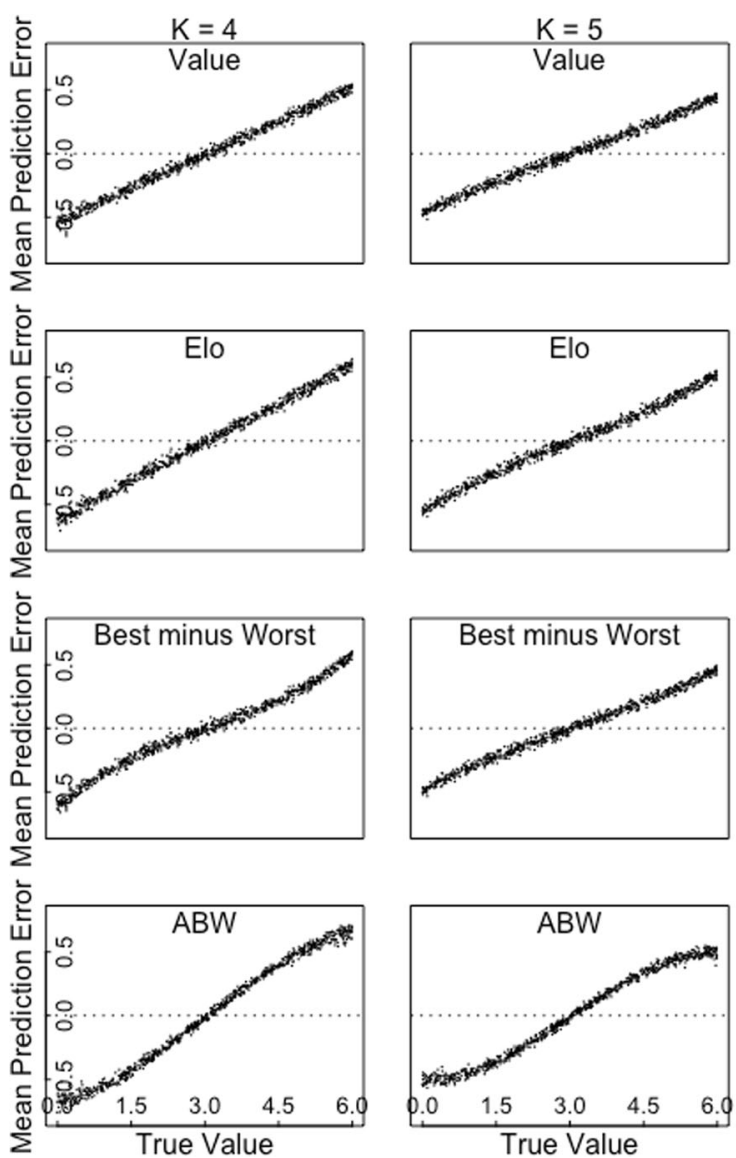

Fig. 17 Magnitudes of errors made by four scoring algorithms when scoring items with uniformly distributed true values. From left to right, the plot columns correspond to $K \mathrm{~s}$ of 4, 5, 6, and 7. From top to bottom, the plot rows correspond to the value, Elo, best-minus-worst, and analytic

The results of note are that the expected inverted-U shape between $K$ and concreteness decision times was observed and that the critical threshold of $K$ again seemed to be higher than 4 . In the case of predicting concrete/abstract decision latencies, all six of the scoring algorithms showed decrements in performance for $K=7$, not just the count-based scoring methods. Also of note is that prediction-based scoring methods outperformed countbased scoring methods for predicting concrete/abstract decision latencies, unlike ELP lexical decision times, for which the opposite was true. $\mathrm{ABW}$ was relatively poorer at predicting the outcome measure in this second set of data.

Estimating word arousal Word arousal norms were collected in addition to word concreteness norms, to provide a highernoise contrastive case. Word arousal judgments are typically more variable than are concreteness judgments, which provided us an opportunity to test for effects of $K$ under different levels of noise in the data. This is desirable to check, since noise level played a clear role in the effects of $K$ from the simulations in Experiment 1.

Arousal judgments were collected for the same 420 words for which concreteness judgments were collected above. As
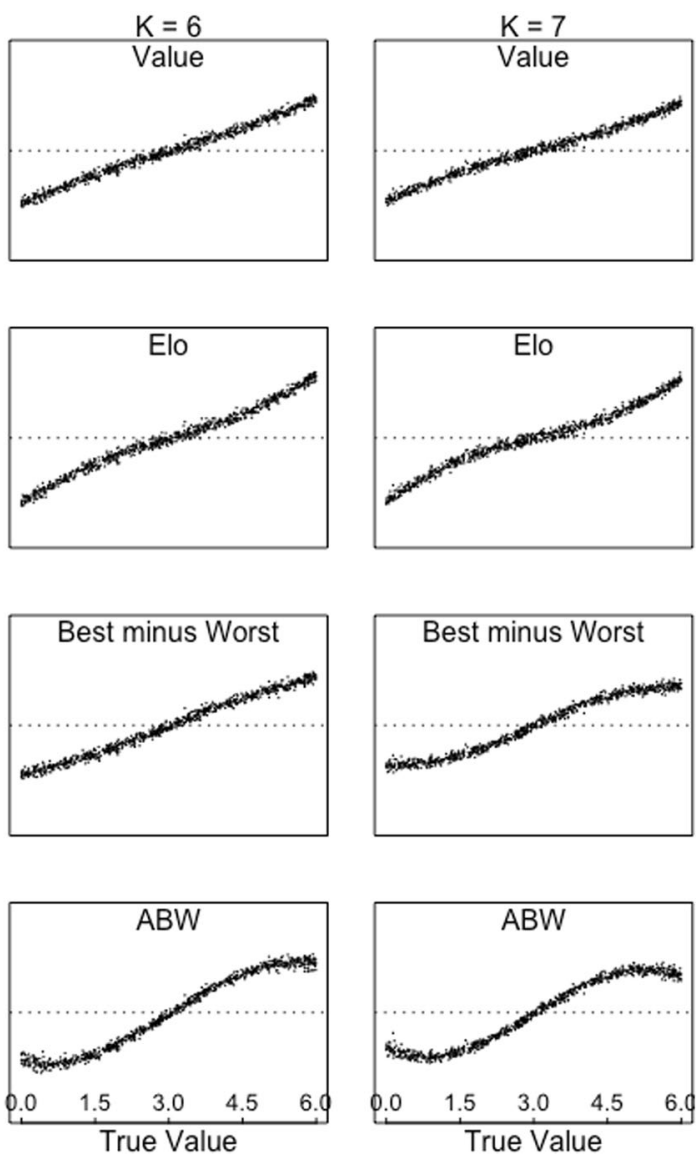

best-worst $(\mathrm{ABW})$ scoring algorithms. Results are shown for the noise condition of 2.0 and are averaged over simulations with numbers of trials from $1 N$ to $32 N$

with the concreteness judgments, the data were subsampled in order to simulate norms for which judgments for $1 \mathrm{~N}, 2 \mathrm{~N}$, or $4 N$ trials' worth of data were collected (100 simulations each), in addition to the full $8 \mathrm{~N}$ of the present study. Correlations of the full data and the subsampled data with the ELP lexical decision times are displayed in Fig. 11.

The quality of fits of the arousal estimates to lexical processing times increases for each increment of $K$ from four items per trial to six items per trial. The quality of the fits drops off when seven items were presented on each trial. This is similar to the patterns observed for concreteness judgments (Fig. 9), with the exception that for concreteness judgments, the decrement in predictive validities for $K=7$ was not present for the prediction-based scoring methods. When estimating word arousal, however, the decrement is present for both predictionbased and count-based scoring methods.

\section{Discussion}

The present experiment replicated the simulation experiments from Experiment 1 with empirical human judgments. In Experiment 1, best-worst scaling estimated the true values 


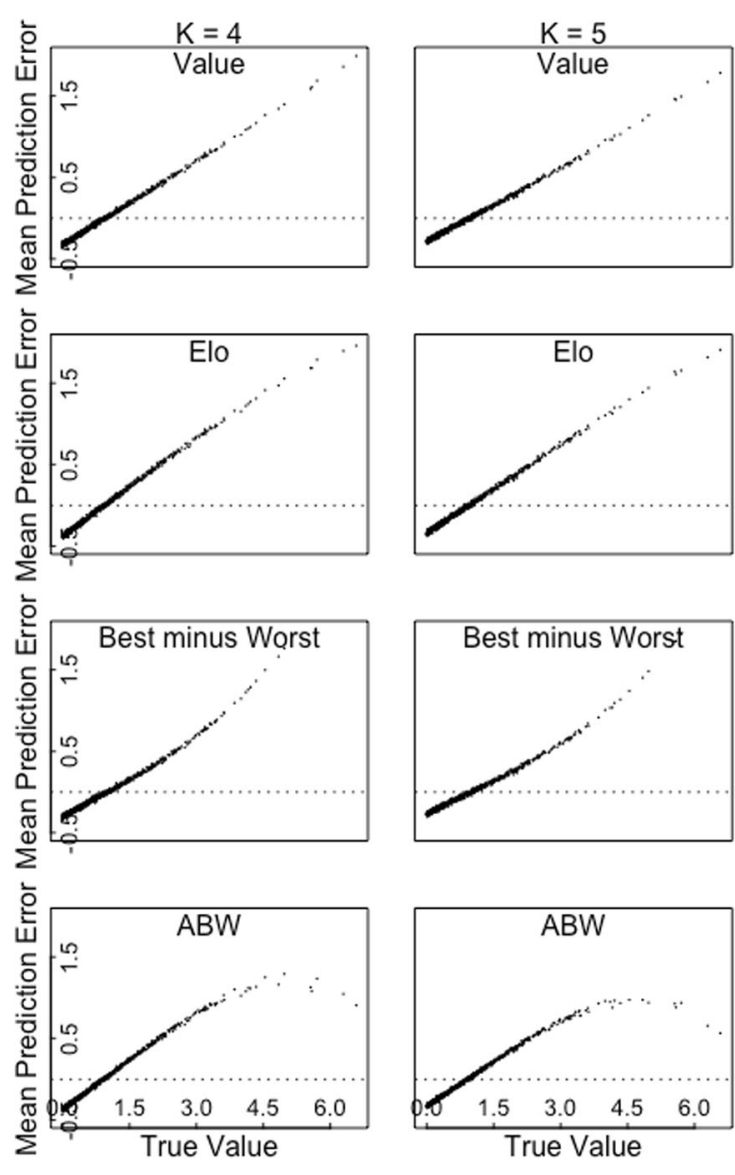

Fig. 18 Magnitude of errors made by four scoring algorithms when scoring items with exponentially distributed true values. From left to right, the plot columns correspond to $K \mathrm{~s}$ of $4,5,6$, and 7. From top to bottom, the plot rows correspond to the value, Elo, best-minus-worst, and

of items better as $K$ was increased, up to some critical value of $K$, at which point the quality of the estimates decreased. The nature of this relationship depended on a variety of factors, including the scoring method used, the number of trials for which data were collected, and the amount of noise present in the simulated judgments.

When examining human judgments, we are unable to compare estimated values to true values, because we simply do not know the true values for the semantic properties of words. Instead, the quality of the norms was measured by using them to predict lexical processing times; insofar as a semantic property of a word plays a role in lexical processing, better estimates of that semantic property should more strongly correlate with measures of lexical processing. When using this methodology to measure the quality of semantic norms produced by best-worst scaling, the empirical results of Experiment 2 are largely consistent with the simulation results of Experiment 1.

The key findings of Experiment 2 are as follows. First, increasing the number of items per trial increases the predictive validity of semantic norms, up to some critical number of items per trial $(K=6)$, at which point the predictive validity of norms is decreased. For arousal judgments, this was seen
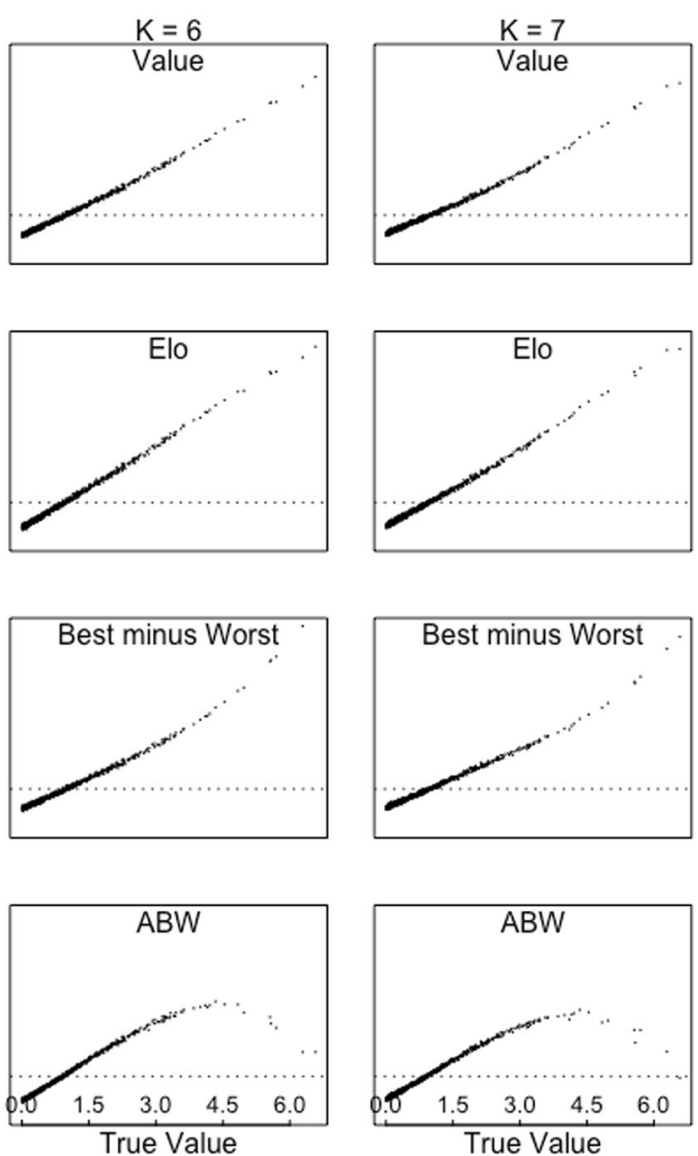

analytic best-worst (ABW) scoring algorithms. Results are shown for the noise condition of 2.0 and are averaged over simulations with numbers of trials from $1 N$ to $32 N$

regardless of the scoring algorithm used. For concreteness judgments, this pattern was seen for count-based scoring methods, but the dip after a critical threshold was not observed for prediction-based scoring methods (though it may have been observed if large enough $K$ s were tested).

We interpret this finding the same way it was interpreted in Experiment 1: It reflects competing forces of more information being made available per trial as $K$ is increased, but that information being increasingly more about relationships to extreme items.

There was a third reason why $K$ was predicted to affect the quality of the estimates produced by best-worst scaling: Specifically, as $K$ increased, participants would be under greater cognitive demands. This should, in turn, decrease the quality of produced estimates at some critical point. We predicted that this effect would manifest as amplifying the decrement in estimate quality for large values of $K$, as compared to the simulation results. However, upon reflection, we are not so confident that the present experiment was designed in such a way as to tease apart the effect of cognitive demand from the informational reasons why the quality of norms would decrease for larger values of $K$. Thus, we simply note that there 

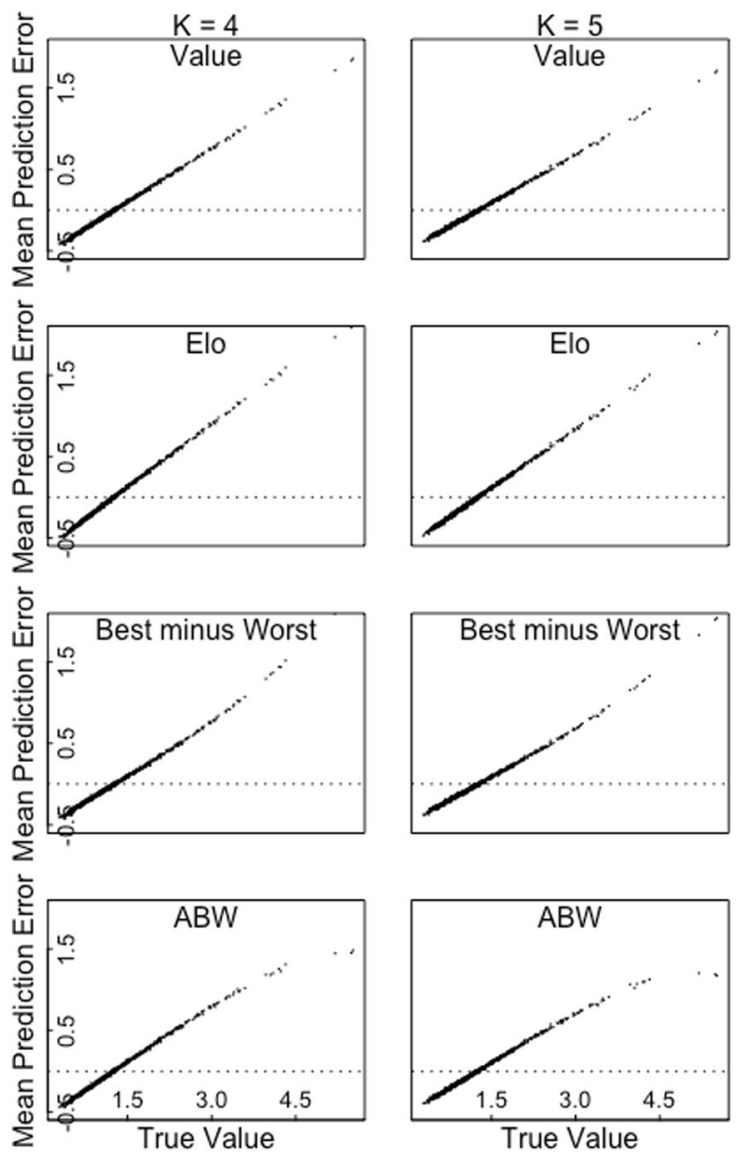

Fig. 19 Magnitudes of errors made by four scoring algorithms when scoring items with $F$-distributed true values. From left to right, the plot columns correspond to $K \mathrm{~s}$ of 4, 5, 6, and 7. From top to bottom, the plot rows correspond to the value, Elo, best-minus-worst, and analytic best-

might be cognitive reasons why we should expect that increasing $K$ beyond some critical threshold would reduce the quality of estimates produced by best-worst scaling, but we are unable to speak to the nature of that relationship currently.

One of the findings from Experiment 1 was that increasing the amount of noise present in judgments also increased the critical value for $K$, beyond which further increases reduced the quality of estimates derived from best-worst scoring. We attempted to validate this simulation result empirically by having participants make judgments for two different semantic dimensions that are known to have different degrees of reliability: Concreteness judgments are more reliable than arousal judgments. The expected finding was that concreteness judgments would show a decrement in predictive validity for large values of $K$, but arousal judgments would not. This pattern was not observed. Both types of judgments showed a decrement in predictive validity for the norms at $K=7$.

Possibly, the difference in reliability between concreteness judgments and arousal judgments was not large enough to produce the expected effect. More specifically, although arousal judgments have low reliability, perhaps that reliability is not
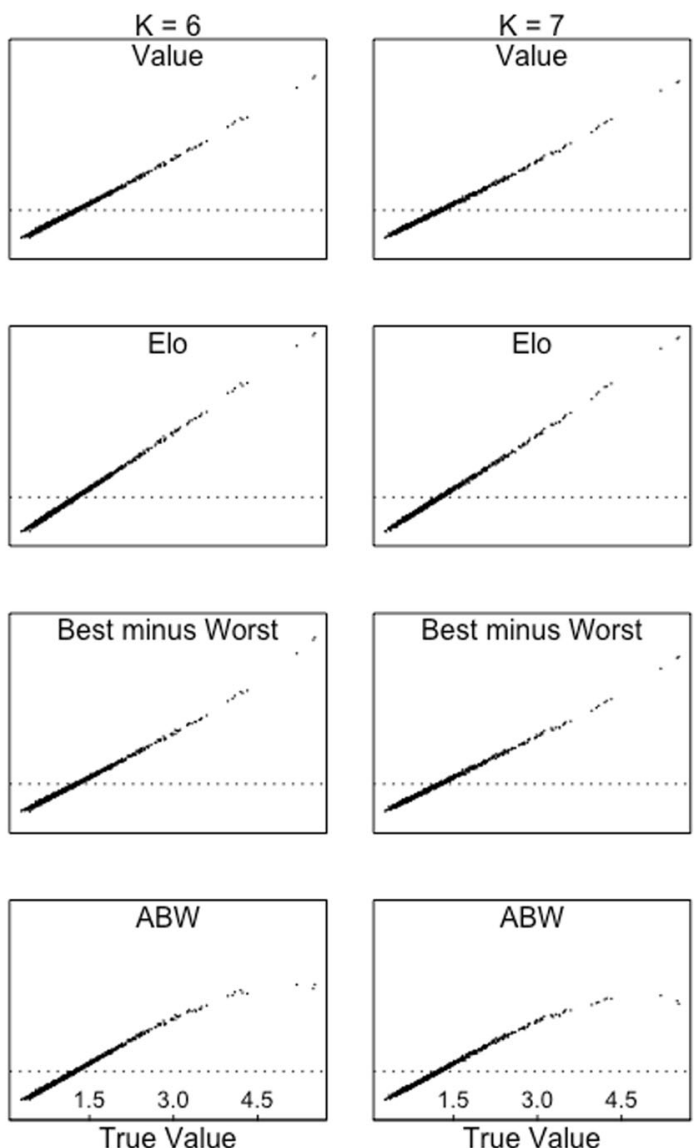

worst (ABW) scoring algorithms. Results are for shown the noise condition of 2.0 and are averaged over simulations with numbers of trials from $1 N$ to $32 N$

low enough to show the effect observed in Experiment 1 . Another option is the nature of variability in empirical versus simulated judgments. Noise was always simulated has having a normal distribution. However, it could be that this does not reflect noise in empirical judgments and that the shape of the distribution of noise matters. We note that most applications of bestworst scaling that employ BIBDs assume that noise is Gumbeldistributed, not normally distributed (Lipovetsky \& Conklin, 2014). This discrepancy between the present simulations and other modeling work using best-worst scaling is addressed further in Appendix 2, where we find that our simulated results do not depend substantially on assumptions about the shape of the noise distribution. Finally, it could also simply be that the model of best-worst scaling used for simulating results in Experiment 1 is an inaccurate or incomplete account of the process of making best-worst decisions and is, thus, misleading on some points.

\section{Conclusion}

This present research was conducted in order to examine the relationship between $K$ and the quality of estimates produced 


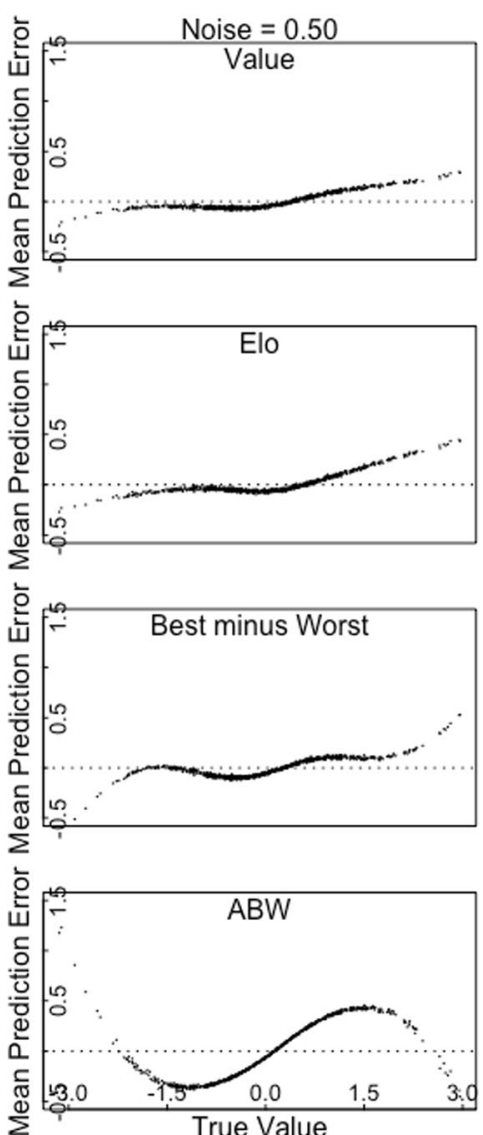

Fig. 20 Magnitudes of errors made by four scoring algorithms when scoring items with normally distributed true values and a Gumbel noise distribution. From left to right, the plot columns correspond to noise standard deviations of $0.5,1.0$, and 2.0. From top to bottom, the plot
Noise $=1.00$ Value
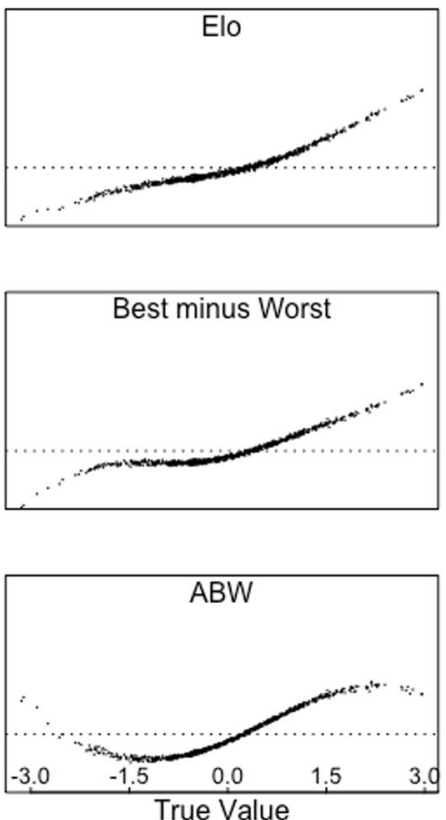

Noise $=2.00$

Value
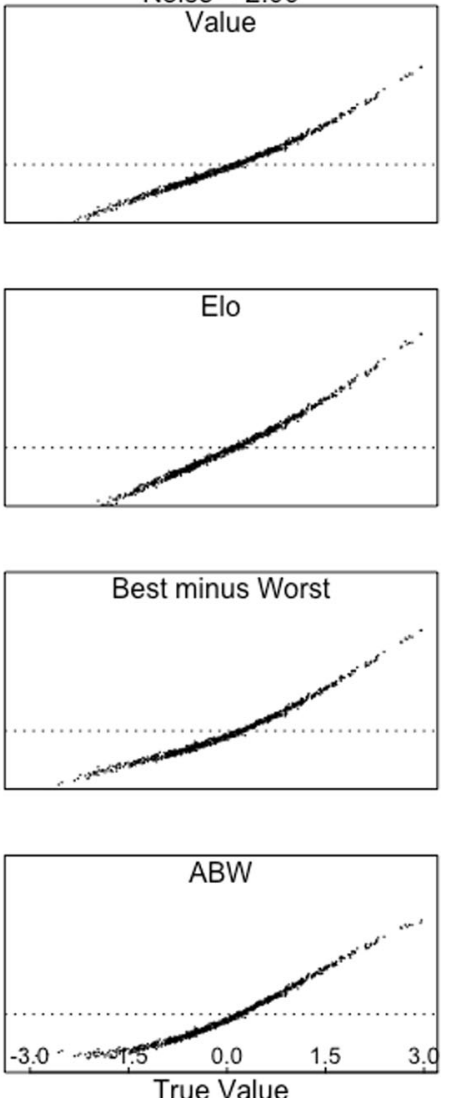

rows correspond to the value, Elo, best-minus-worst, and analytic bestworst (ABW) scoring algorithms. Results are shown for a $K$ of 6 and are averaged over simulations with numbers of trials from $1 N$ to $32 N$

by best-worst scaling. The results of Experiments 1 and 2 strongly suggest that in the general case, the preferred value of $K$ in many- $N$ best-worst scaling experiments is not 4 . We recommend that researchers use $K=6$ if they have little knowledge about the underlying distribution of true values or the amount of noise that will be present in their data. Simulation results suggest that smaller $K$ s may be preferred with less-noisy data and larger $K$ s may be preferred with more-noisy data. This is particularly the case for count-based scoring algorithms (but see the next paragraph). We note, however, that empirical results do not support these conclusions about noise-dependent choices in $K$. This may simply be due to a restricted range in the variability of human judgments tested in Experiment 2.

Hollis and Westbury (2018) suggested that $8 N$ trials balances well the cost of data collection effort with the accuracy of estimates. No strong evidence in the present results suggests otherwise, although we note that this is a parameter that still needs further exploration.

We present some ancillary results that provide rough guidance concerning which scoring algorithms to prefer in bestworst scaling experiments. Most important is that the scoring algorithm that will best fit some criterion measure, whether it be true values or a behavioral measure of cognitive processing, depends substantially on multiple factors: at least on $K$, the amount of noise present, the number of trials data are collected for, the underlying distribution of true values, or the type of behavioral measure that a fit is being made to. Best-minus-worst scoring has almost exclusively been used in many- $N$ applications of best-worst scaling. We strongly suggest that researchers consider applying the other scoring algorithms, as well; they all can be applied to the same data. At a pragmatic level, this helps ensure that researchers are getting the most (predictive) value out of their collected data. At a theoretical level, this will help build up a better understanding of the variety of types of errors that best-worst scoring algorithms make, and under which conditions.

Acknowledgments Harald Baayen and 2 anonymous reviewers are thanked for their generous and helpful critiques on the presentation and relevance of the vector learning model. Jason Hicks' pointed guidance as editor played an essential role in carving this manuscript out of the muck that was its prior versions. This manuscript would have never come to fruition without years of discussion on naive discriminative learning with Chris Westbury. 


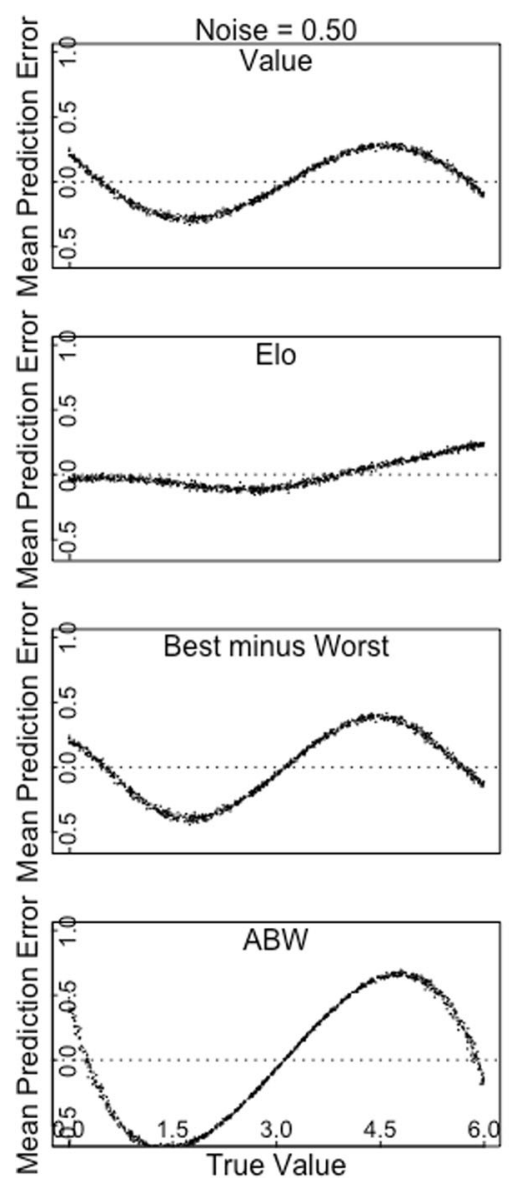

Fig. 21 Magnitudes of errors made by four scoring algorithms when scoring items with uniformly distributed true values and a Gumbel noise distribution. From left to right, the plot columns correspond to noise standard deviations of $0.5,1.0$, and 2.0. From top to bottom, the
Noise $=1.00$ Value
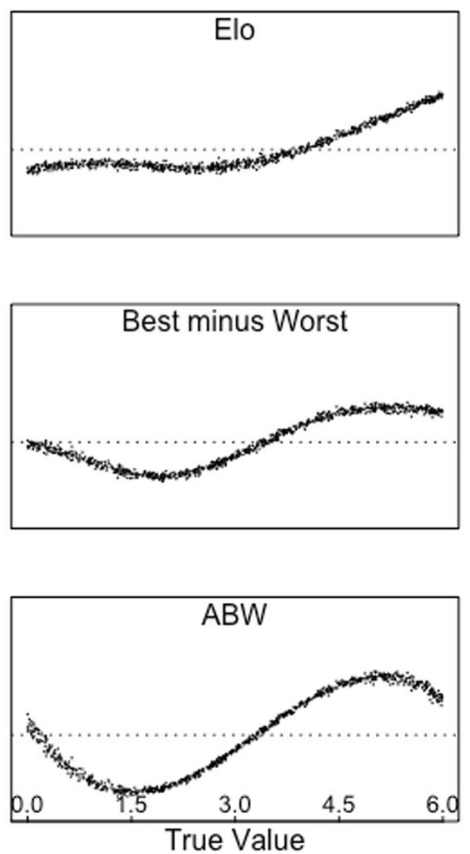

Noise $=2.00$ Value
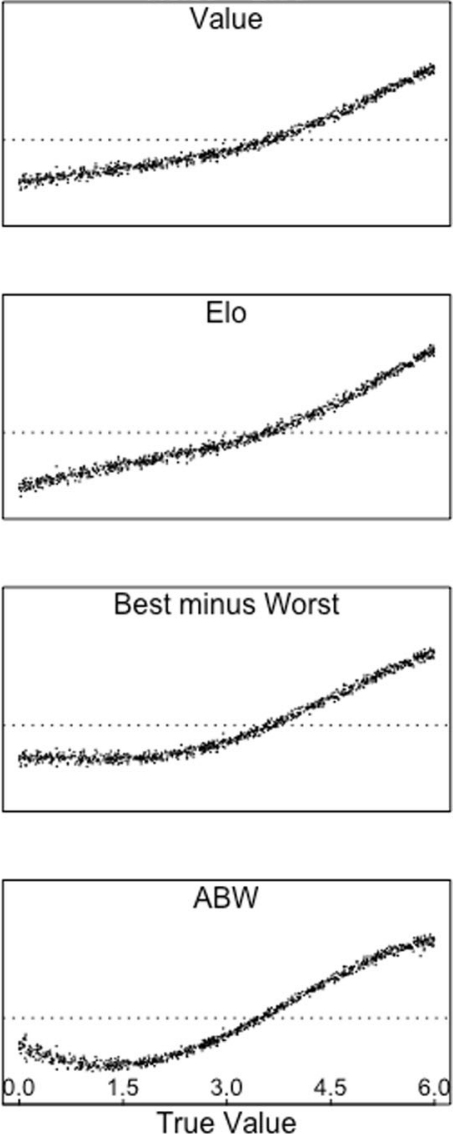

plot rows correspond to the value, Elo, best-minus-worst, and analytic best-worst (ABW) scoring algorithms. Results are shown for a $K$ of 6 and are averaged over simulations with numbers of trials from $1 N$ to $32 N$

\section{Appendix 1}

This appendix contains replots of Figs. 4, 5, 6, and 7 with noise levels of 1 and $2 S D$ s rather than $0.5 S D$ (Figs. 12, 13, 14 , and 15 and $16,17,18$, and 19 , respectively). The main thing to note is that as noise levels increase, the pattern of prediction errors made by all scoring algorithms tend to converge. Notable deviations are that the prediction errors tend to be more extreme for Elo than for the other scoring algorithms for high noise levels, whereas errors at extreme values are relatively less pronounced for high noise levels.

\section{Appendix 2}

The present research is based on the assumption that when noise is normally distributed across the latent dimension of interest. A normal distribution of noise is a common assumption when modelling psychological processes and is supported in the case of comparative judgment by Thurstone (1927). However, most applications of best-worst scaling assume that judgment noise has a Gumbel distribution. When using BIBDs, best-minus-worst scores and ABW scores can be demonstrated to be unbiased estimators of item utilities as derived by the multinomial logit model if noise is Gumbeldistributed (Lipovetsky \& Conklin, 2014).

Within a constrained set of cases (Gumbel-distributed noise, use of BIBDs), both best-minus-worst scores and $\mathrm{ABW}$ scoring have a formal motivation for their use. However, it is unclear how the two scoring methods would be expected to behave outside of those cases. In Experiment 1 's simulations, neither BIBDs were used nor noise was modeled with a Gumbel distribution. Thus, perhaps the simulation results were unfairly disadvantaging best-minus-worst and ABW scoring. This is a relevant concern, considering that ABW did outperform the other scoring algorithms on at least one of the empirical validations from Experiment 2.

The use of BIBDs is of course not feasible in large- $N$ designs. However, it is possible to simulate results using different distributions of noise. The purpose of this appendix is to reevaluate the various scoring algorithms when noise is assumed to be Gumbel-distributed rather than normally distributed. 


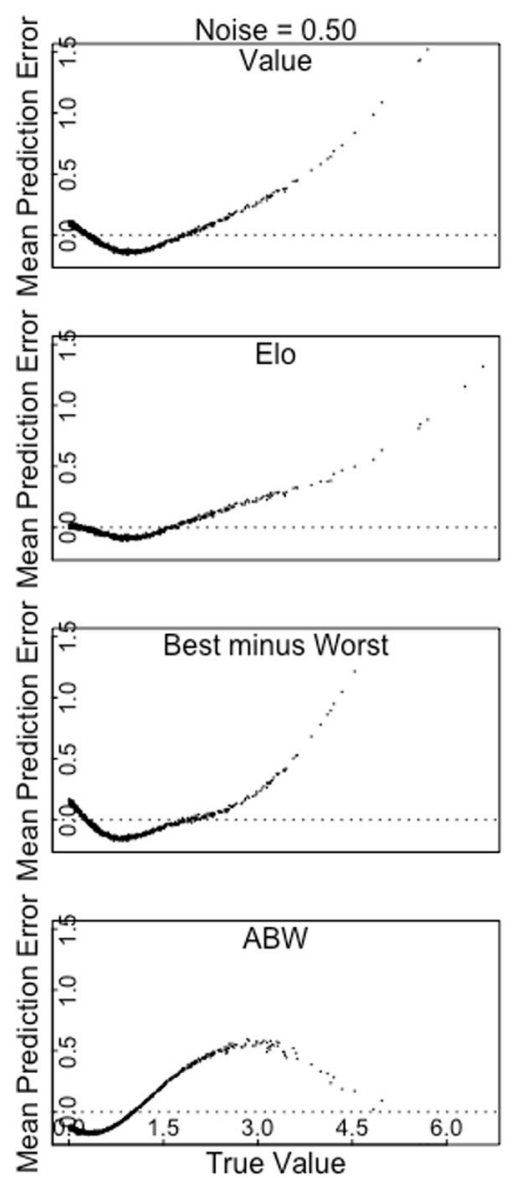

Fig. 22 Magnitudes of errors made by four scoring algorithms when scoring items with exponentially distributed true values and a Gumbel noise distribution. From left to right, the plot columns correspond to noise standard deviations of $0.5,1.0$, and 2.0. From top to bottom, the plot rows
Noise $=1.00$
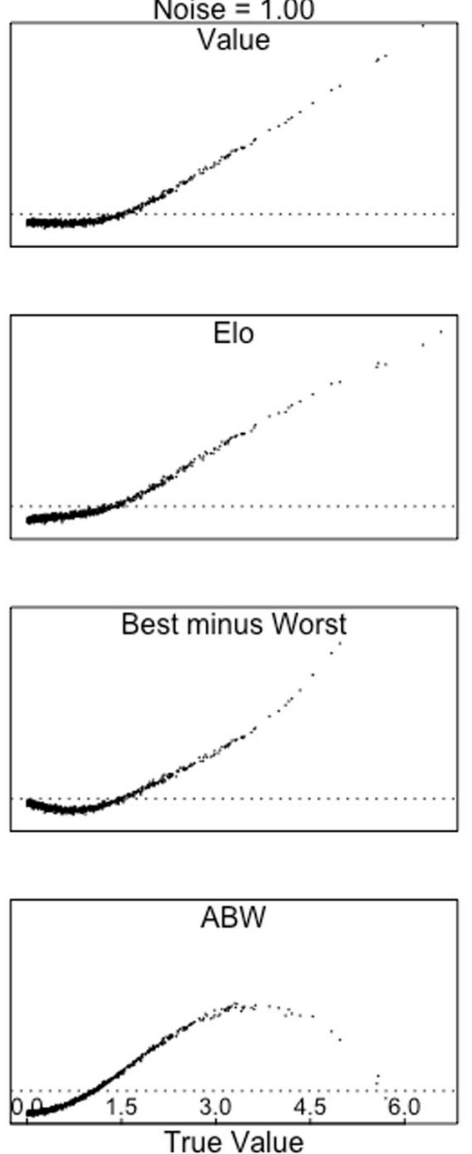

Noise $=2.00$
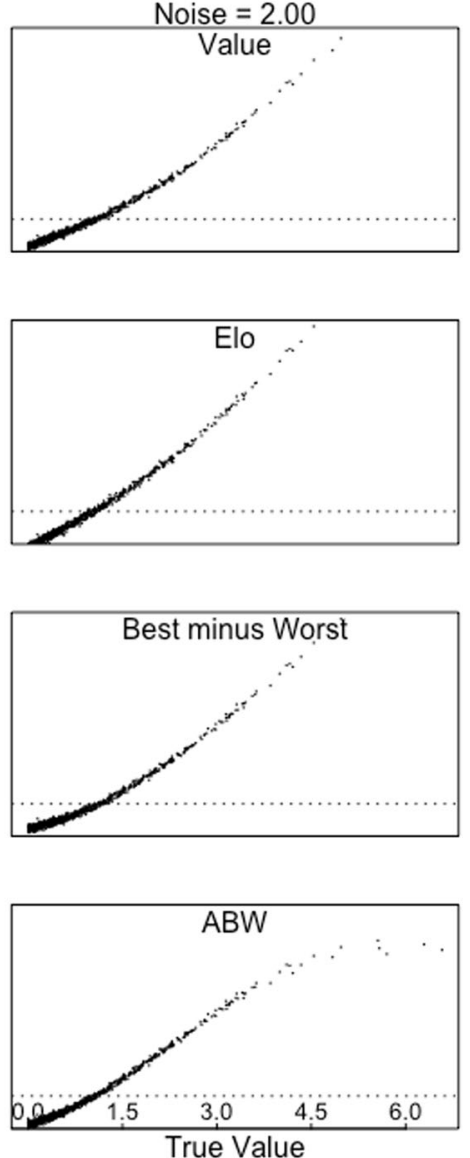

correspond to the value, Elo, best-minus-worst, and analytic best-worst (ABW) scoring algorithms. Results are shown for a $K$ of 6 and are averaged over simulations with numbers of trials from $1 N$ to $32 N$

\section{Method}

All methodological details were the same as in Experiment 1, with the following exceptions: Judgment noise was simulated as being Gumbel-distributed (standard deviations of $0.5,1.0$, and 2.0), and only the case of $K=6$ was tested. We tested only one case of $K$ because of the computational cost of running this simulation. Choosing a $K$ of 6 is justified on the basis of the results of experiments 1 and 2 .

\section{Results}

Two indices of performance were evaluated: degree of bias in estimates of the true values, and overall fit, measured in terms of $R^{2}$, between the estimates and true values. Estimate biases are plotted similarly to Figs. 4, 5, 6, and 7 from Experiment 1: Estimates were regressed on the true values and error is plotted for each item, averaged across independent simulations. The results are displayed in Figs. 20, 21, 22, and 23, for normal, uniform, exponential, and $F$-distributed true values, respectively. As with Experiment 1, only the results for value,
Elo, best-minus-worst, and ABW scores are presented. The error profiles for Rescorla-Wagner scoring were similar to those for value scores, and David scores were similar to best-minus-worst scores. The main thing to note is that all of the scores, including best-minus-worst and ABW, are biased estimates of the true values for all levels of noise tested. Unbiased estimates would be indicated by scatter around a horizontal line with an intercept of 0 . The bias profiles vary by scoring method at low noise levels, but begin to converge for high levels of noise. This is similar to what was observed in Experiment 1 when noise was simulated to be normally distributed.

The second measure of performance we report is $R^{2}$ values between the estimates and true values. The results are presented in Table 1. For the purpose of comparison, fits for Gumbel and normal noise distributions with similar standard deviations are presented side by side. The largest increase in fit for assuming Gumbeldistributed noise is seen for ABW scoring when the true values are normally distributed and noise has a standard deviation of 0.5 . In this case, the fit improved from $R^{2}$ 


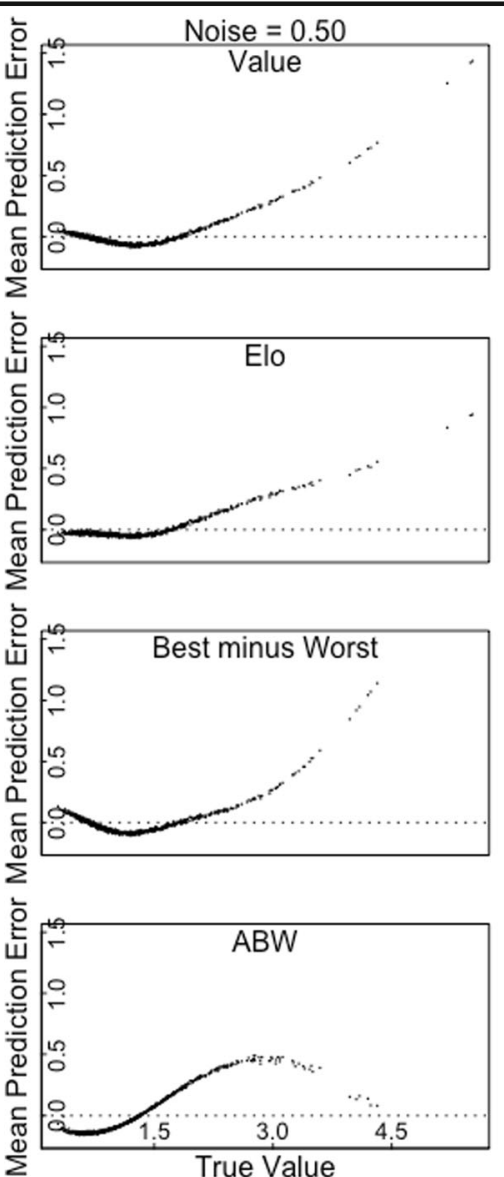

Fig. 23 Magnitudes of errors made by four scoring algorithms when scoring items with $\mathrm{F}$ distributed true values and a Gumbel noise distribution. From left to right, the plot columns correspond to noise standard deviations of $0.5,1.0$, and 2.0. From top to bottom, the plot
Noise $=1.00$ Value
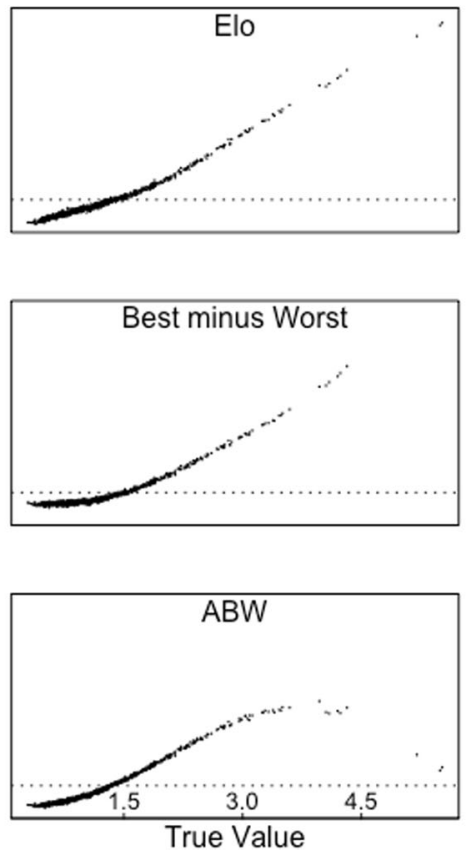

rows correspond to the value, Elo, best-minus-worst, and analytic bestworst (ABW) scoring algorithms. Results are shown for a $K$ of 6 and are averaged over simulations with numbers of trials from $1 N$ to $32 N$

estimators of true values. In fact, none of the scoring methods is without some bias. This was an observation also noted in Experiment 1. When examining $R^{2}$ rather than measures of bias, ABW does show a small increase in fit for data that have Gumbel-distributed rather than normal-distributed noise in some cases (notably, normally distributed true values with low levels of noise). However, this increase is small, and ABW was outperformed in that same case by all of the other scoring algorithms it was compared against.

The general pattern of results observed in Experiment 1 does not substantially depend on assuming normally distributed rather than Gumbel-distributed noise. The estimation biases observed in $\mathrm{ABW}$, best-minus-worst scoring, and all of the other scoring algorithms likely are the result of not being able to employ BIBDs in large- $N$ applications of best-worst scaling. Although the relative performance of the scoring algorithms depends on the distribution of the true values, it does not appear to depend on the noise distribution of the judgments. 
Table 1 Fits $\left(R^{2}\right)$ between true values and score estimates under different simulation conditions

\begin{tabular}{|c|c|c|c|c|c|c|c|}
\hline \multirow[b]{3}{*}{ Latent distribution } & \multicolumn{7}{|c|}{ Noise distribution } \\
\hline & \multirow[t]{2}{*}{ No noise } & \multicolumn{3}{|c|}{ Gumbel } & \multicolumn{3}{|c|}{ Normal } \\
\hline & & 0.50 & 1.00 & 2.00 & 0.50 & 1.00 & 2.00 \\
\hline \multicolumn{8}{|l|}{ Normal } \\
\hline Value & .95 & .93 & .86 & .74 & .93 & .88 & .73 \\
\hline Rescorla-Wagner & .95 & .93 & .85 & .73 & .93 & .87 & .72 \\
\hline Elo & .97 & .92 & .83 & .68 & .93 & .86 & .67 \\
\hline Best-Worst & .94 & .92 & .85 & .74 & .93 & .87 & .72 \\
\hline $\mathrm{ABW}$ & .73 & .80 & .81 & .73 & .76 & .79 & .70 \\
\hline David & .92 & .93 & .85 & .74 & .92 & .86 & .72 \\
\hline \multicolumn{8}{|l|}{ Uniform } \\
\hline Value & .90 & .94 & .92 & .86 & .94 & .93 & .87 \\
\hline Rescorla-Wagner & .89 & .94 & .91 & .85 & .94 & .93 & .87 \\
\hline Elo & .95 & .95 & .91 & .83 & .96 & .94 & .85 \\
\hline Best-Worst & .90 & .95 & .92 & .85 & .93 & .92 & .86 \\
\hline $\mathrm{ABW}$ & .58 & .82 & .85 & .82 & .80 & .85 & .83 \\
\hline David & .88 & .92 & .91 & .85 & .92 & .92 & .87 \\
\hline \multicolumn{8}{|l|}{ Exponential } \\
\hline Value & .78 & .88 & .82 & .70 & .89 & .86 & .72 \\
\hline Rescorla-Wagner & .80 & .90 & .83 & .70 & .91 & .87 & .72 \\
\hline Elo & .79 & .90 & .80 & .64 & .92 & .86 & .67 \\
\hline Best-Worst & .76 & .83 & .79 & .69 & .86 & .84 & .72 \\
\hline ABW & .64 & .82 & .79 & .71 & .83 & .79 & .72 \\
\hline David & .77 & .85 & .80 & .69 & .86 & .83 & .71 \\
\hline \multicolumn{8}{|l|}{$F$} \\
\hline Value & .82 & .87 & .77 & .61 & .89 & .81 & .63 \\
\hline Rescorla-Wagner & .84 & .88 & .77 & .61 & .90 & .82 & .63 \\
\hline Elo & .82 & .87 & .73 & .52 & .90 & .79 & .54 \\
\hline Best-Worst & .80 & .83 & .75 & .61 & .85 & .80 & .63 \\
\hline $\mathrm{ABW}$ & .69 & .81 & .76 & .62 & .82 & .77 & .64 \\
\hline David & .81 & .84 & .75 & .60 & .85 & .80 & .63 \\
\hline
\end{tabular}

All cell values are averaged over 100 independent simulations of the parameter setting

\section{References}

Auger, P., Devinney, T. M., \& Louviere, J. J. (2007). Using bestworst scaling methodology to investigate consumer ethical beliefs across countries. Journal of Business Ethics, 70, 299-326.

Balota, D. A., Yap, M. J., Cortese, M. J., Hutchison, K. A., Kessler, B., Loftis, B., . . Treiman, R. (2007). The English Lexicon Project. Behavior Research Methods, 39, 445-459. https://doi.org/10.3758/ BF03193014

Bradley, M. M., \& Lang, P. J. (1999). Affective norms for English words (ANEW): Stimuli, instruction manual and affective ratings (Technical Report C-1). Gainesville, FL: University of Florida, NIMH Center for Research in Psychophysiology.

Brysbaert, M., Warriner, A. B., \& Kuperman, V. (2014). Concreteness ratings for 40 thousand generally known English word lemmas. Behavior Research Methods, 46, 904-911. https://doi.org/10.3758/ s13428-013-0403-5

David, H. A. (1987). Ranking from unbalanced paired-comparison data. Biometrika, 74, 432-436.
Elo, A. E. (1973). The International Chess Federation rating system. Chess, 38(July), 293-296; 38(August), 328-330; 39(October), 19 21.

Finn, A., \& Louviere, J. J. (1992). Determining the appropriate response to evidence of public concern: The case of food safety. Journal of Public Policy and Marketing, 12-25.

Hollis, G. (2018). Scoring best-worst data in unbalanced many-item designs, with applications to crowdsourcing semantic judgments. Behavior Research Methods, 50, 711-729. https://doi.org/10.3758/ s13428-017-0898-2

Hollis, G., \& Westbury, C. (2018). When is best-worst best? A comparison of best-worst scaling, numeric estimation, and rating scales for collection of semantic norms. Behavior Research Methods, 50, 115-133. https://doi.org/10.3758/ s13428-017-1009-0

Hollis, G., Westbury, C., \& Lefsrud, L. (2017). Extrapolating human judgments from skip-gram vector representations of word meaning. Quarterly Journal of Experimental Psychology, 70, 1603-1619. https://doi.org/10.1080/ 17470218.2016.1195417

Jurgens, D., Mohammad, S., Turney, P., \& Holyoak, K. (2012). Semeval2012 task 2: Measuring degrees of relational similarity. In Proceedings of the 6th International Workshop on Semantic Evaluation (pp. 356-364). Stroudsburg, PA: Association for Computational Linguistics.

Keuleers, E., Lacey, P., Rastle, K., \& Brysbaert, M. (2012). The British Lexicon Project: Lexical decision data for 28,730 monosyllabic and disyllabic English words. Behavior Research Methods, 44, 287-304. https://doi.org/10.3758/ s13428-011-0118-4

Kiritchenko, S., \& Mohammad, S. M. (2016, June). Capturing reliable fine-grained sentiment associations by crowdsourcing and best-worst scaling. Paper presented at the 2016 Annual Conference of the North American Chapter of the Association for Computational Linguistics: Human Language Technologies (NAACL), San Diego, CA.

Kiritchenko, S., \& Mohammad, S. M. (2017). Best-worst scaling more reliable than rating scales: A case study on sentiment intensity annotation. In Proceedings of the Annual Meeting of the Association for Computational Linguistics (pp. 465-470). Stroudsburg, PA: Association for Computational Linguistics.

Kiritchenko, S., Zhu, X., \& Mohammad, S. M. (2014). Sentiment analysis of short informal texts. Journal of Artificial Intelligence Research, 50, 723-762.

Kuperman, V., Estes, Z., Brysbaert, M., \& Warriner, A. B. (2014). Emotion and language: Valence and arousal affect word recognition. Journal of Experimental Psychology: General, 143, 1065-1081. https://doi.org/10.1037/a0035669

Lipovetsky, S., \& Conklin, M. (2014). Best-worst scaling in analytical closed-form solution. Journal of Choice Modelling, 10, 60-68.

Louviere, J. J., Flynn, T. N., \& Marley, A. A. J. (2015). Best-worst scaling: Theory, methods and applications. Cambridge, UK: Cambridge University Press.

Marley, A. A. J., Islam, T., \& Hawkins, G. E. (2016). A formal and empirical comparison of two score measures for best-worst scaling. Journal of Choice Modelling, 21, 15-24.

Paivio, A. (2013). Imagery and verbal processes. Hove, UK: Psychology Press.

Pexman, P. M., Heard, A., Lloyd, E., \& Yap, M. J. (2017). The Calgary Semantic Decision Project: Concrete/abstract decision data for 10 , 
000 English words. Behavior Research Methods, 49, 407-417. https://doi.org/10.3758/s13428-016-0720-6

Soutar, G. N., Sweeney, J. C., \& McColl-Kennedy, J. R. (2015). Bestworst scaling: An alternative to ratings data. In J. J. Louviere, T. N. Flynn, \& A. A. J. Marley (Eds.), Best-worst scaling: Theory, methods and applications (pp. 177-188). Cambridge, UK: Cambridge University Press.

Thurstone, L. L. (1927). A law of comparative judgment. Psychological Review, 34, 273-286. https://doi.org/10.1037/ h0070288

Westbury, C. F., Shaoul, C., Hollis, G., Smithson, L, Briesemeister, B. B., Hofmann, M. J., \& Jacobs, A. M. (2013). Now you see it, now you don't: On emotion, context, and the algorithmic prediction of human imageability judgments. Frontiers in Psychology, 4, 991. https://doi. org/10.3389/fpsyg.2013.00991

Open Practices Statement All software used in this research is available at the author's website, www.ualberta.ca/ hollis. The data for Experiment 2 will be made available by request to the author.

Publisher's note Springer Nature remains neutral with regard to jurisdictional claims in published maps and institutional affiliations. 